Version: June 1, 2022

\title{
V2492 CYGNI: THE EARLY EVOLUTION OF THE 2010 OUTBURST
}

\author{
Colin Aspin $^{1}$
}

\begin{abstract}
We present Gemini-North optical and near-IR observations of a young eruptive star in Cygnus, designated as V2492 Cygni in the General Catalog of Variable Stars. This object is one of two young stars, located within 2 degrees of each other, that recently brightened by around 5 mags and were reported as possible new FU Orionis-type variables.

The outburst spectrum of V2492 Cygni shows atomic emission features throughout the optical and near-infrared. In the optical, $\mathrm{H} \alpha$ is in emission and has an associated blue-shifted absorption component. The far-red Ca II triplet lines are also in emission and, as with $\mathrm{H} \alpha$, possess significant blue-shifted absorption. The optical $\mathrm{TiO}$ molecular bands are also in emission. In the near-infrared, $\operatorname{Pa} \beta, \operatorname{Br} \gamma$, and the $\mathrm{CO}$ overtone bandheads are strongly in emission. Such spectral characteristics are very similar to those exhibited by both EX Lupi, the progenitor of the EXor class of eruptive variables, during its 2008 extreme outburst, and V1647 Ori during its elevated phase in 2003.

Additionally, we consider archival data on V2492 Cygni and investigate the pre-outburst nature of this young star. We construct a quiescent-phase spectral energy distribution which, via model fitting, gives insight into the circumstellar environment of the object prior to the current eruption.

Our investigation strongly suggests that the V2492 Cygni outburst appears to be more consistent with an EXor event, a fact supported by its recent 2 mag fading and stochastic variability.
\end{abstract}

Subject headings: stars: individual (V2492 Cygni) — circumstellar matter stars: formation

\footnotetext{
${ }^{1}$ Institute for Astronomy, University of Hawaii at Manoa, 640 N. A'ohoku Place, Hilo, HI 96720. caa@ifa.hawaii.edu,reipurth@ifa.hawaii.edu
} 


\section{INTRODUCTION}

During the month of August 2010, eruptive outbursts of the two young stars HBC 722 and V2492 Cygni were reported by Semkov \& Peneva (2010a,b) and Itagaki (2010), respectively. Both stars are located in the extensive Cygnus star formation complex near the North America and Pelican nebulae (see Reipurth \& Schneider 2008 and references therein), and both were found to have optically brightened by between 4 and 6 magnitudes. In the case of HBC 722, this brightening occurred over a period of around three months, while for V2492 Cyg, the change in brightness occurred over 10 months. V2492 Cyg was independently discovered by the Palomar Transient Factor (PTF) and labeled PTF10nvs in a detailed study by Covey et al. (2011). The American Association of Variable Star Observers, the AAVSO, designate the star VSX J205126.1+440523.

These two independent events are significant because such large amplitude outbursts are rare occurrences. Two types of eruptive events are known in young stellar objects (YSOs), specifically, those named after FU Orionis (Wachmann 1954; Herbig 1966) and those named after EX Lupi (Herbig 1977). FU Orionis outbursts, often referred to as FUor outbursts (Ambartsumian 1971), show 5-6 magnitude increases in optical brightness and can take from decades to even a century to fade back into quiescence. The two most most recent FUor events observed were the outbursts of V1057 Cyg in 1969 (Welin 1971; Herbig \& Harlan 1971) and the eruption of V1647 Ori in 2004 (e.g., Reipurth \& Aspin 2004, Briceno et al. 2004, Aspin \& Reipurth 2009). The latter has had an unusual light curve, fading away after only two years, but returning again to its elevated state, and today the available evidence supports its classification as a FUor. EX Lupi outbursts, commonly called EXors (Herbig 1977, 1989), exhibit optical brightness increases of 2-5 mags and fade after periods of a few months to a few years (Herbig 1977, 2008). They are also observed to be repetitive. For example, the EXor variable VY Tau brightened and faded more than a dozen times between 1930 and 1970 (Meinunger 1969, 1971; Herbig 1977, 1990).

Both types of eruptive events are thought to be driven by the same underlying process, commonly believed to be enhanced mass accretion through a disturbed/disrupted circumstellar disk and onto the young stellar photosphere. Quiescent $\mathrm{T}$ Tauri star accretion rates of $10^{-7}$ to $10^{-8} \mathrm{M}_{\odot} \mathrm{yr}^{-1}$ have been observed to increase by a factor of 100 to 1000 during eruptive outbursts and can thereby result in a significant amount of mass being accreted over relatively short timescales. This so-called "accretion disk model" (Hartmann \& Kenyon 1985, 1996) can explain many of the observed properties of both FUors and EXors, although the mechanism by which the disk becomes unstable is still being debated. An outstanding question regarding FUor and EXor outbursts is why they differ so much in many of their observed characteristics including, for example, the event timescale and amplitude, and their 
optical and near-infrared (NIR) spectral properties (FUors exhibit predominantly optical and NIR absorption spectra whereas EXors always possess strong emission features). An alternative explanation for FUor outbursts has been proposed by Herbig (1989), Herbig et al. (2003), and Petrov \& Herbig $(1992,2008)$. Their model, which is based on a rapidly rotating star with regions of significantly different photospheric temperatures, can also explain many of the observed characteristics of FUor eruptions.

In this paper we present optical and NIR observations of the recently discovered eruptive variable V2492 Cyg. The star is located in a small molecular cloud core surrounded by a bright rim in the Lynds Bright Nebula LBN 343. Our goals are to i) characterize the early stages of the eruption, ii) better understand how such a young star fits into the established framework of FUor and EXor outbursts, iii) provide new observations that can be used to further test theoretical/numerical models, and $i v$ ) gain further insight into the initiation and progression of such dramatic and highly energetic events.

\section{OBSERVATIONS AND DATA REDUCTION}

Table 1 shows the complete observing log for the observations presented below. All observations of V2492 Cyg were taken using the "Fredrick C. Gillett" Gemini-North 8 m telescope located on Mauna Kea, HI, in September, October, and November 2010 (see Table 1 for complete dates). The instrument used for the optical observations was GMOS-N, the facility optical imager and spectrograph (Davies et al. 1997; Hook et al. 2004). For the imaging, we used the r' filter and a total exposure time per source of $60 \mathrm{~s}$. For the spectroscopy, we used the B600 grating and central wavelength $7500 \AA$. A 0 .'75 wide long-slit was used resulting in a resolving power, $R$, of $\sim 1200\left(0.45 \AA \mathrm{pixel}^{-1}\right)$. This value of $R$ gives a full-width half maximum (FWHM) of unresolved lines of $\sim 130 \mathrm{~km} \mathrm{~s}^{-1}$. The total on-source exposure time for the spectroscopy was $300 \mathrm{~s}$. Identical observations of the spectrophotometric standard star G191-B2B were also taken to allow flux calibration and sensitivity function definition. All spectra were reduced using the Gemini GMOS iraf package (v1.10).

Our NIR imaging observations were taken over the aforementioned period using the Gemini-North facility NIR camera, NIRI (Hodapp et al. 2003). Due to the intrinsic brightness of the sources, the $\mathrm{f} / 32$ camera was used (allowing shorter exposure times) and data was acquired in J, H, K', and L' filters. For each filter, four dithered images were taken to allow for both sky subtraction and uncertainty estimation. Total exposure times of $20 \mathrm{~s}$ were used for J, H, and K', and 48 s for L'. Similar observations of the NIR faint standard star FS 150 were also taken for flux calibration purposes. The images were reduced using the Gemini NIRI iraf package (v1.10). Extraction of aperture photometry from the reduced 
images was performed using the Starlink gaia program.

NIR spectroscopic observations were acquired using the Gemini-North facility integralfield unit (IFU) spectrograph, NIFS (McGregor et al. 2003). Observations using the J, H, and $\mathrm{K}$ gratings were taken with total exposure times of $120 \mathrm{~s}$ per waveband and resulted in spectra with a spectral resolution of $R \sim 5000$. Sky observations, taken using an 'ABBA' offset sequence, were also acquired to facilitate accurate sky subtraction. Similar observations of the A0 V star HIP 103222 were taken to allow the removal of telluric features from the target spectra. The data were reduced using the Gemini NIFS iraf package (v1.10). The final spectra of the targets are the sum of the IFU pixels lying within an 0.5 radius software aperture centered on the targets. Flux calibration was performed using the broad-band J, H, and $\mathrm{K}$ source brightness close to the time of the spectroscopic observations. We converted the broad-band magnitudes to flux density using the Gemini Observatory conversion tool].

\section{V2492 Cyg AND ITS ENVIRONMENT}

Below we consider our knowledge of V2492 Cyg prior to its 2010 outburst. In what follows, we use the phrase 'post-outburst' to refers to the period from immediately after the rise to maximum light but prior to the sources return to its pre-outburst, quiescent brightness.

\subsection{At Optical Wavelengths}

In optical archival images taken in October 1997, V2492 Cyg appears as a faint, unremarkable star located close to the edge of an irradiated molecular cloud edge around $15^{\prime}$ southeast of the Pelican Nebula (see Figure 1). It is associated with an IRAS source, IRAS 20496+4354, and is the possible exciting source of a Herbig-Haro bow shock, HH 569, some $2^{\prime}(0.35 \mathrm{pc})$ to the south (Bally \& Reipurth 2003). Images of the region immediately surrounding V2492 Cyg are shown in Figure 2, Bally \& Reipurth (2003) classified IRAS $20496+4354$ as a moderate-luminosity Class I protostellar object. In the Dobashi et al. (2005) visual extinction $\left(\mathrm{A}_{V}\right)$ maps, V2492 Cyg lies near a filamentary dust structure in a region with a general line-of-sight $A_{V}$ of $\sim 4-5$ mags. The distance to the North America/Pelican Nebula region of Cygnus has been estimated to be $550 \pm 50$ pc (Laugalys et al.

\footnotetext{
${ }^{1}$ http://www.gemini.edu/sciops/instruments/midir-resources/imaging-calibrations/fluxmagnitudeconversion
} 
2006).

Itagaki (2010) recently documented eruptive behavior in V2492 Cyg finding that its optical (unfiltered) brightness rose from $>17.5$ on UT 2008 Aug 7 to 13.8 two years later. The commencement of this outburst, however, could have been any time between UT 2008 Aug 7 and his next observation on UT 2009 Dec 19. His multiple observations between 2009.9 and 2010.7 show a close to linear rise in optical brightness of approximately $0.25 \mathrm{mag} \mathrm{month}^{-1}$. For reference, USNO-B1 gave optical magnitudes for V2492 Cyg of B=20.17 and R=18.28 on 1979.9. Covey et al. (2011) presented an extensive, detailed study of V2492 Cyg including considerable R-band optical photometry from the Palomar Transient Factory images. These data demonstrated that the current event peaked in August 2010 (at $\mathrm{R}=13.3$ ) and rather than the linear rise in brightness as seen by Itagaki (2010), significant stochastic variability was present from as early as August 2009. In addition, their light curve showed multiple brightness peaks with a typical peak-to-peak timescale of $\sim 100$ days suggesting the possibility of periodic as well as uncorrelated variability.

\subsection{At Infrared Wavelengths}

V2492 Cyg was not detected in the 2MASS survey at any wavelength. This implies that at the time the survey images were acquired, the source was considerably fainter than the quoted $2 M A S S 10 \sigma$ detection limits of $15.8,15.1$, and 14.3 mags at $\mathrm{J}, \mathrm{H}$, and $\mathrm{K}$ ', respectively (Skrutskie et al. 2006)2. We have, however, found a detection of V2492 Cyg in the UKIDSS3 database (Lawrence et al. 2007) from UT 2006 June 10; the only wavelength at which it is brighter than the $2 M A S S$ detection limits is $\mathrm{K}$ ' where it has a magnitude of $\sim 13.3$. A comparison of the $2 M A S S$ and UKIDSS K' images (taken in 2000 and 2006, respectively) is shown in Figure 3. Magnier et al. (1999) included V2492 Cyg in a catalog of young stellar objects (YSOs) and noted that at far-IR wavelengths it had a flat spectrum suggestive of an object transitioning from the protostellar to $\mathrm{T}$ Tauri evolutionary phase. Finally, Covey et al. (2011) present NIR photometric observations from five epochs spanning the period July 2010 to September 2010.

The AKARI/IRC Point Source Catalog (Murakami et al. 2007) contains a source within $6^{\prime \prime}$ of the optical/NIR coordinates with 9 and $18 \mu \mathrm{m}$ fluxes of 2.0 and $2.9 \mathrm{Jy}$, respectively. The

\footnotetext{
${ }^{2}$ Figure 15 of Skrutskie et al (2006) indicates that the $3 \sigma$ detection limit of $2 M A S S$ is approximately 0.5 , 1.0 , and 1.4 mags fainter at $\mathrm{J}, \mathrm{H}$, and $\mathrm{K}$, respectively. In the $2 M A S S$ images, there is no indication of any object at the optical/NIR coordinates of V2492 Cyg

${ }^{3}$ UKIDSS is the UKIRT Infrared Deep Sky Survey. See http://www.UKIDSS.org.
} 
star was not detected in AKARI/FIS observations. Spitzer did, however, detect V2492 Cyg in all four IRAC bands and with MIPS at 24 and $70 \mu \mathrm{m}$. Spitzer images, centered on V2492 Cyg, are shown in Figure 4. MSX6C also detected V2492 Cyg at infrared wavelengths from 8.28 to $21.34 \mu \mathrm{m}$.

The IRAS source associated with V2492 Cyg has Point-Source Catalog (PSC) fluxes at $12,25,60$, and $100 \mu \mathrm{m}$ fluxes of 3.4, 6.6, 28.2, and $58.3 \mathrm{Jy}$, respectively. The precessed IRAS coordinates are within $0{ }^{\prime \prime} 5$ of the optical/NIR position and therefore well within the $16^{\prime \prime}$ IRAS error ellipse. No obvious optical/infrared sources are in the immediate vicinity of V2492 Cyg, making the association of optical and IRAS sources firm. One interesting point, however, is that the IRAS $60 \mu \mathrm{m}$ flux (taken in 1983) is $5 \times$ larger than the $60 \mu \mathrm{m}$ Spitzer/MIPS flux (taken in 2006/2007).

\subsection{At $\mathrm{mm}$ Wavelengths}

The Bolocam Galactic Plane Survey (GPS) contains $1.1 \mathrm{~mm}$ images of the region containing V2492 Cyg and lists a source within 4" of its coordinates (GPS designation G084.46600.135). The flux quoted in the GPS catalog is $148 \pm 74 \mathrm{mJy}$ and since the next closest $\mathrm{mm}$ source is $46^{\prime \prime}$ away, we adopt this value for its $1.1 \mathrm{~mm}$ flux.

\section{RESULTS}

\subsection{Optical Imaging}

The optical images of V2492 Cyg presented in Figure 2 show a pre-outburst $1997 \mathrm{H} \alpha$ image taken from Bally \& Reipurth (2003, left) and our post-outburst Gemini GMOS r' image (right) taken on UT 2010 September 5. V2492 Cyg, located at the image centers, is very much brighter post-outburst. No obvious optical nebulosity is seen around V2492 Cyg, even in its photometrically elevated state. Nor was one found in the R-band images of Covey et al. (2011). For comparison, we note that all classical FUors and FUor-like objects have been found to exhibit nebulous structure in outburst which often takes the form of curving tails extending from the young star (Goodrich 1985). EXors do not typically possess associated nebulosity.

Due to source saturation, we could not obtain photometry from our optical images, however, we note that at the time of the GMOS observations, the American Association of Variable Star Observers (AAVSO) database shows the source having an optical V-band 
magnitude of $15.1 \pm 0.1$. In Figure 5 we show the AAVSO V-band light curve of V2492 Cyg. In addition to the AAVSO data 4 , we have also included the 'unfiltered' photometry from Itagaki (2010, blue downward-facing arrows) and the R-band data from Covey et al. (2011, grey filled circles). The Itagaki (2010) photometry serves as an indication of the optical progression of the rise from quiescence to outburst and since these data are in unfiltered optical light, they are upper limits. The Covey et al. (2011) data indicates both the level of variability present over the time period shown.

\subsection{Near-Infrared Photometry}

We can place V2492 Cyg in NIR color-color (henceforth c-c) diagrams and thereby investigate the level and associated variability of overlying extinction and intrinsic thermal excess emission. One note of caution is, however, warranted. If V2492 Cyg is a Class I protostar, its NIR fluxes may be significantly modified by circumstellar scattering. This was studied in detail by Whitney, Kenyon, \& Gomez (1997) who, using simple extinction+scattering models, discussed the effect of scattering on emergent flux. Below we assume that the direct flux from V2492 Cyg is not dominated by scattering but that it is true, reddened stellar/disk flux. Our results are likely not applicable if scattered flux forms the major component of the observed NIR signal.

Figure 6a shows on the a NIR J-H vs. H-K (henceforth JHK) while Figure 6b shows the $\mathrm{H}-\mathrm{K}$ vs. $\mathrm{K}-\mathrm{L}$ (henceforth HKL) c-c diagram. Some of the 2 and $3 \mu \mathrm{m}$ photometric data included were taken using filters with slightly different central wavelengths and bandpasses (e.g. K and K', and L and L'), however, within the associated uncertainties (typically $\sim 10 \%$ on photometry, $\sim 14 \%$ on colors) we consider the slight differences in magnitudes/fluxes between filters unimportant for the analysis below. Our new NIR photometric values are detailed in Table 2 .

In the JHK c-c diagram (panel a), points \#1, \#4, and \#8 shows the location of V2492 Cyg at three different epochs i.e. the UKIDSS (JHK') observations (UT 2006 June 10) and our two Gemini/NIRI (JHK'L') observations (UT 2010 September 5 and November 25). In our Gemini/NIRI data from September 5 (the closest observation to the peak optical brightness), V2492 Cyg has a K' magnitude of 8.12 \pm 0.1 while approximately 3 months later (November 25) it had faded to $9.63 \pm 0.1$. With a pre-outburst UKIDSS K' magnitude of 13.15, the source had therefore brightened at $2 \mu \mathrm{m}$ by $\sim 5$ mags between 2006 (\#1) and 2010

\footnotetext{
${ }^{4}$ We refer the reader to the AAVSO web site at http://www.aavso.org for access to the V-band photometry shown in Figure 5 .
} 
(\#4). This is likely the result of a combination of a decrease in $2 \mu \mathrm{m}$ thermal excess and/or a reduction in overlying visual extinction 5 In addition to points $\# 1$, \#4, and \#8, we have also included the J, H, and K photometry from Covey et al. (2011), points \#2, \#3, and $\# 5$, \#6, and \#7, in chronological order. The trend from the earliest photometry (\#1) to the observation just after maximum brightness (\#4) is approximately linear with both $\mathrm{J}-\mathrm{H}$ and $\mathrm{H}-\mathrm{K}$ decreasing with time. This progression is close to being along a reddening vector suggesting that the change is due to a reduction in overlying extinction. In Figure 6a we show reddening vectors (dashed lines) using the extinction law defined by Robitaille et al. (2007) and used in their young star spectral energy distribution (SED) models. These extend from the extremes of the zero-age main sequence/giant branch and from the end of the CTTS locus defined by Greene \& Meyer (1995), the latter extends between points labeled A and B. It may be the case that the linear change in location of V2492 Cyg in the JHK c-c diagram is the result of the presence of a somewhat different reddening law than that shown in Figure 6a. Perhaps more plausibly, the variation in JHK colors could be caused by a combination of changes in overlying extinction and K-band thermal excess.

To explore the first of these two options, we plot a selection of reddening laws together with the V2492 Cyg data in Figure 7 with the sequence of JHK observation of V2492 Cyg shown as filled black circles. Point \#1, however, is outside the area plotted but is located on the dashed green line (see Figure 6a for details). All reddening vectors, together with the dotted line extending to point \#1, originate at the red end of the CTTS locus (point B, again, see Figure 6a). We have plotted four different reddening laws defined in the literature and often used in young star JHK diagrams. All vectors represent $\mathrm{A}_{V}=10$ mags. The solid grey lines show the Cardelli, Clayton, and Mathis (1989, henceforth CCM) reddening vectors for four different values of $\mathrm{R}$, the ratio of total to selective extinction. The values shown are for $\mathrm{R}=2,3.1,5.0$, and 7.0. $\mathrm{R}=3.1$ is the typical interstellar reddening value while $\mathrm{R}=5.0$ is often quoted for extinction towards young stars in dense dark clouds. As can be seen, all four CCM reddening vectors have very similar slopes with the major effect of changing the value of $\mathrm{R}$ being the length of the $A_{V}=10$ mags reddening vector. Close to the CCM vectors lies a generic reddening law in which the wavelength dependence of reddening is proportional to the scaled wavelength (with respect to the value at $0.55 \mu \mathrm{m}$ ) to the power of $\alpha=-1.6$ (red dashed line). Reddening from this relationship closely matches the CCM reddening laws. The single-dotted-dash line (blue) shows the Rieke \& Lebofsky (1985) reddening law defined using observations of the galactic center while the triple-dotted-dash line (purple) shows the law adopted by Robitaille et al. (2007) in the generation of their SED models. The latter

\footnotetext{
${ }^{5}$ An alternative may be that the disk is hotter at maximum and optically thick gas interior of the dust sublimation radius adds NIR continuum flux.
} 
two reddening laws produce somewhat different slopes with respect to the CMM and $\alpha=-$ 1.6 laws. However, none of these reddening laws extend through the sequence of V2492 Cyg colors. What we can conclude from Figure 7 is that neither changes in the adopted reddening law nor in the value of $\mathrm{R}$ used would result in the colors variations observed in V2492 Cyg.

The location of the three Covey et al. (2011) JHK observations, obtained between our two Gemini/NIRI datasets (i.e. points \#5-7), indicate that within a few days of our acquisition of the data that gave rise to point \#4 the source's JHK colors began to reverse the progression observed (from points \#1 to \#4). A similar behavior was seen during the 2003-2006 eruption of V1647 Ori (Aspin, Beck, \& Reipurth 2008) where it was attributed to a variation of overlying extinction of around 10 mags. However, in the case of the color variations of V1647 Ori, they occurred directly along a Rieke \& Lebofsky (1985) reddening vector. Our most recent observation, point \#8, shows that this reversal continued and at the end of November 2010 the source's JHK colors were similar to what they were in July 2010 (points \#2 and \#3) although in November 2010, V2492 Cyg had a K' magnitude of $9.63 \pm 0.1$ while in July 2010 Covey et al. found a value of $8.45 \pm 0.02$. As an exercise, if we attribute the total change in JHK colors from point \#2 to point \#4 to changes in $\mathrm{A}_{V}$, then the amount of overlying visual extinction would have to have reduced by $\sim 7$ mags. If we also attribute the extremely large colors in June 2006 (point \#1) to reddening, then the change of $\mathrm{A}_{V}$ from $\# 1$ to $\# 4$ would amount to $\sim 20$ mags.

In our HKL c-c diagram (Figure $6 \mathrm{~b}$, right) there exists only two epochs of data. Point \#4 (2010 September 5) shows V2492 Cyg to possess a significant $3 \mu \mathrm{m}$ thermal excess suggesting that even though the outburst had already occurred, a considerable amount of heated dust must still be present in the circumstellar environment close to the star. Approximately three months later (UT 2010 November 25, point \#8), the $3 \mu \mathrm{m}$ thermal excess had grown even larger implying a continuing build-up of dust close enough to the star/accretion region to be heated to temperatures of $\sim 1000 \mathrm{~K}$. This dust, however, must be far enough so as not to be sublimated at temperatures of $\sim 1500 \mathrm{~K}$. Tuthill et al. (2001), and subsequently Lykou et al. (2010), suggested that the distance from a star where dust sublimates can be estimated using the equation

$$
r_{\text {sub }}=1.1 \times\left(T_{\text {sub }} / 1500 K\right)^{-2} \times\left(L_{\text {bol }} / 1000 L_{\odot}\right)^{0.5}
$$

where $\mathrm{r}_{\text {sub }}$ is the sublimation radius in $\mathrm{AU}, \mathrm{T}_{\text {sub }}$ is the sublimation temperature in $\mathrm{K}$, and $\mathrm{L}_{b o l}$ is the system bolometric luminosity in $\mathrm{L}_{\odot}$. Assuming $\mathrm{T}_{\text {sub }}=1500 \mathrm{~K}$ and a preoutburst $\mathrm{L}_{b o l}$ of $\sim 35 \mathrm{~L}_{\odot}$ (Bally \& Reipurth 1997; Covey et al. 2011), we obtain a value for $\mathrm{r}_{\text {sub }}$ of $\sim 0.2 \mathrm{AU}$. In its elevated state, Covey et al. (2010) suggest that the bolometric luminosity increases by a factor 100. This would result in $\mathrm{r}_{\text {sub }}$ increasing by a factor 10 to 
$\sim 2$ AU. Therefore, we suggest that the dust content of the disk region between 0.2 and 2 AU must have been accreted or sublimated during the outburst.

\subsection{Spitzer Photometry}

Similarly, c-c plots can be made from Spitzer IRAC and MIPS photometry. Such diagrams are useful for assigning evolutionary classes to young stars since in them Class I protostars, Class II classical T Tauri stars (CTTSs), Class III weak-line T Tauri stars, and main sequence stars are well separated (Allen et al. 2002). We have extracted photometric magnitudes from the Spitzer IRAC and MIPS images using the IDL routine photvis (v1.1, Gutermuth et al. 2004, 20086). These values are given in Table 5, In the IRAC [3.6]-[4.5] vs. [5.6]-[8.0] plot (Figure 8a) V2492 Cyg (point \#1) lies well within the Class I regime. In the IRAC/MIPS [3.6]-[4.5] vs. [8.0]-[24] plot (Figure 8b), the source lies on the Class I-II boundary. In these Figures we also show the locations of several FUors for comparative purposes (points \#2 through \#6) together with the location of V2492 Cyg using the Spitzer fluxes given in Covey et al. (2011, point \#7). What we can conclude from these plots is that the location of V2492 Cyg is consistent with it being a Class I protostar. We note that in the [3.6]-[4.5] vs. [5.6]-[8.0] plot, V2492 Cyg lies closest to the FUor-like object L1551 IRS5 (point \#5)7. We are uncertain as to why the Covey et al. (2011) [3.6]-[4.5] vs. [8.0]-[24] V2492 Cyg point is displaced from our measurement since both use the same datasets. Perhaps this indicates that the uncertainties on the extracted $24 \mu \mathrm{m}$ photometry are somewhat larger than the error bars shown.

\subsection{Optical Spectroscopy}

\subsubsection{The UT 2010 September 5 GMOS-N Spectrum}

Our GMOS-N optical spectrum of V2492 Cyg from UT 2010 September 5 is shown in Figure 9 (top). It has a signal-to-noise of $\sim 200$ and was acquired close to maximum optical brightness. The spectrum is heavily veiled and, hence, no photospheric absorption features are visible. V2492 Cyg shows a wealth of emission lines including H $\alpha$ (6563 ^), Ca II (8498, 8542, and $8662 \AA$ ), and numerous neutral and singly ionized Fe lines. Both K I (7665 and

\footnotetext{
${ }^{6}$ We have used the same aperture sizes and photometric zeropoints as in the aforementioned papers.

${ }^{7}$ L1551 IRS5 was saturated in the Spitzer MIPS $24 \mu \mathrm{m}$ images and so our photometry is that given at $24 \mu \mathrm{m}$ in Osorio et al. (2003).
} 
$7699 \AA$ ), and O I (7773 and $8446 \AA$ ) are, however, in absorption. We note that Li I is not detected. The complete list of emission and absorption lines with equivalent widths, EW, $\geq 1 \AA$, together with flux values, are given in Table 3. Figure 9 (bottom) also shows a comparison of this optical spectrum with that of an M6 V dwarf. As Covey et al. (2011) discovered, and we confirm here, the $\mathrm{TiO}$ bands, found in absorption in late-type stars, are in emission in V2492 Cyg. The spectrum of the M6 V dwarf is used here as an example of a late-type star showing strong $\mathrm{TiO}$ band absorption and we have marked the extent of the TiO emission in V2492 Cyg by horizontal lines above the M6 V spectrum.

Although the resolving power of our GMOS spectra is only $R \sim 1200$, we can nevertheless obtain some insight into the velocity structure present in various spectral features via line profile fitting. We note that an unresolved arc lamp line using the B600 grating and 0'.75 slit has a FWHM $130 \mathrm{~km} \mathrm{~s}^{-1}$ and, hence, profile fitting should conservatively give reliable velocity information at the $\sim 20 \%$ level or $\sim \pm 30 \mathrm{~km} \mathrm{~s}^{-1}$. It is this value we adopt for the uncertainty on quoted velocities.

The $\mathrm{H} \alpha$ emission feature shows associated blue-shifted absorption out to velocities of $\sim-450 \mathrm{~km} \mathrm{~s}^{-1}$, similar to that found by Covey et al. (2011). The Ca II triplet emission lines possess weak blue-shifted absorption components out to velocities of $\sim-300 \mathrm{~km} \mathrm{~s}^{-1}$. The absorption components on $\mathrm{H} \alpha$ and $\mathrm{Ca}$ II can be better seen in the expanded views shown in Figure 10, $\mathrm{H} \alpha$ has an EW of $-16.3 \AA$ but in reality the value will be somewhat larger (i.e. more negative) due to the presence of the associated absorption components. The (deconvolved) full-width 10\% intensity (FW10\%) of $\mathrm{H} \alpha$ is $550 \mathrm{~km} \mathrm{~s}^{-1}$ while the location of the minimum of the absorption component is $-210 \mathrm{~km} \mathrm{~s}^{-1}$. The peak of the $\mathrm{H} \alpha$ emission is close to zero velocity as it is for the Fe I lines and the Ca II triplet lines. The velocity of the minimum blue-shifted absorption associated with the Ca II triplet lines is $\sim-190 \mathrm{~km} \mathrm{~s}^{-1}$ and, within the associated uncertainties, is the same as on $\mathrm{H} \alpha$.

\subsubsection{The UT 2010 November 25 GMOS-N Spectrum}

Optical spectroscopy of V2492 Cyg was also obtained on Gemini-North using GMOS on UT 2010 November 25. The same instrumental setup was used as for the September 5 spectrum. Figure 11 shows this spectrum in the same format as the earlier spectrum. At the time of acquisition, V2492 Cyg had optically faded by 4.5 mags and had an R-band magnitude of $18 \pm 0.2$ (Covey 2010, private communication).

There are several differences between the optical spectra taken on UT 2010 September

5 and November 25, specifically, the $\mathrm{TiO}$ emission bands have slightly changed shape (as 
was also seen by Covey et al. 2011), H $\alpha$ has lost its deep blue-shifted absorption component, and several other spectral lines have changed strength or have appeared/disappeared. The fading of V2492 Cyg from R 13.5 to 18.0 between UT 2010 September 5 and November 25 would correspond to an EW increase for constant line emission of $\sim 60 \times$. With this in mind, we find that:

- As noted above, $\mathrm{H} \alpha$ has lost its deep blue-shifted absorption component. Absorption appears to be present nonetheless but at lower velocities. One possible scenario is that $\mathrm{H} \alpha$ emission has filled in the blue-shifted absorption. The EW of the $\mathrm{H} \alpha$ emission is -27.6 Aas opposed to $-16.3 \AA$ on September 5. For constant $\mathrm{H} \alpha$ emission, the change in continuum level between the two dates would result in an $\mathrm{H} \alpha \mathrm{EW}$ of $\sim 1000 \AA$. We can conclude that the $\mathrm{H} \alpha$ emission has declined in intensity by a factor $\sim 35$ between the two observations. In addition, the $\mathrm{FW} 10 \%$ intensity of $\mathrm{H} \alpha$ has declined from $550 \mathrm{~km} \mathrm{~s}^{-1}$ to $300 \mathrm{~km} \mathrm{~s}^{-1}$.

- The [O I] lines at 6300 and $6363 \AA$ have become much more prominent and rival H $\alpha$. Their EW changed from -1.3 and $-0.6 \AA$ on September 5 to -38.9 and $-13.3 \AA$ on UT 2010 November 25. This increase in EW is a factor of about 30 suggesting that the [O I] emission declined by around a factor 2 between the two dates. However, the O I absorption lines at 7774 and $8446 \AA$ have weakened by $\sim$ factor 2 over the same time period.

- The reappearance of [S II] lines at 6716 and $6731 \AA$, which were absent in the September 5 spectrum yet present in the optical spectra of Covey et al. (2011), suggests that shocks have reappeared in the intervening period. The ratio of the EWs of the two [S II] emission lines $(6716 / 6731)$ is $\sim 0.6$. Since the production of these lines and their flux ratio are only weakly dependent on temperature and more strongly dependent on density, we have used the IRAF program nebular.temden to calculate the electron density, $n_{e}$, necessary to produce this ratio. We find that a high density environment is needed with an $n_{e} \sim 4000 \mathrm{~cm}^{-3}$ for an electron temperature of $\sim 10^{4} \mathrm{~K}$. We note that the critical density for $[\mathrm{S} \mathrm{II}]$ is $\sim 5 \times$ larger than this value and that typical electron densities in Herbig-Haro shocks is $\sim 1000 \mathrm{~cm}^{-3}$.

- The disappearance of blue-shifted absorption on the Ca II triplet line at $8498 \AA$ is peculiar especially since it is still present and well detected on the 8543 and $8662 \AA$ Ca II triplet lines. On September 5, this line, however, had the weakest blue-shifted absorption of the three lines (see Figure 10). The velocity of the minimum in the blueshifted absorption has declined from $-190 \mathrm{~km} \mathrm{~s}^{-1}$ to $-125 \mathrm{~km} \mathrm{~s}^{-1}$, a proportionally similar velocity change as the blue-shifted absorption on $\mathrm{H} \alpha$. Interestingly, the $\mathrm{EW}$ of 
the Ca II triplet lines are similar on the two observing dates. This implies that as the broad-band R flux declined the Ca II emission declined by a similar amount.

- The K I absorption lines at 7665 and $7699 \AA$ behave in a similar manner to the Ca II triplet lines in that their EW remained approximately constant in the September and November spectra.

\subsection{NIR Spectroscopy}

\subsubsection{The UT 2010 October 2/3 NIFS Spectra}

The NIFS J, H, and K spectra of V2492 Cyg taken on UT 2010 October 2 (K band) and 3 ( $\mathrm{J}$ and $\mathrm{H}$ bands) are shown in Figure13. The full spectral range observed is shown in the topleft panel with the $\mathrm{J}, \mathrm{H}$, and $\mathrm{K}$ bands shown in the top-right, bottom-left, and bottom-right panels, respectively. For comparison we also show the prototypical EXor eruptive variable EX Lup 8 Apart from the continuum slope, which increases with wavelength in V2492 Cyg and decreases with wavelength in EX Lup, almost all features observed in EX Lup are reproduced in V2492 Cyg and are totally dissimilar to those seen in FUors such as FU Ori. Specifically, the CO overtone bandheads are strongly in emission as are $\mathrm{Br} \gamma$ and $\mathrm{Pa} \beta$. Other H I emission lines are also present as are emission lines from $\mathrm{Mg} \mathrm{I}, \mathrm{Na}$ I, and Fe $] 9$. We additionally note that the broad water vapor bands at $1.4 \mu \mathrm{m}$ and $1.8 \mu \mathrm{m}$ are weakly in absorption in EX Lup while in V2492 Cyg they appear weakly in emission (as indicated by the small increase in continuum slope near those wavelengths).

The observed $\mathrm{Pa} \beta$ and $\mathrm{Br} \gamma$ line fluxes in the UT 2010 October $2 / 3 \mathrm{NIR}$ spectra of V2492 Cyg are $1.1 \times 10^{-13}(\mathrm{EW}=-11.8 \AA)$ and $9.0 \times 10^{-14} \operatorname{ergs~cm}^{-2} \mathrm{~s}^{-1} \AA^{-1}(\mathrm{EW}=-5.1 \AA)$, respectively. These correspond to line luminosities of $\sim 0.001 \mathrm{~L}_{\odot}$ in both $\mathrm{Pa} \beta$ and $\operatorname{Br} \gamma$ assuming $\mathrm{A}_{V}=0, \mathrm{~d}=600 \mathrm{pc}$, and that the line flux is emitted isotropically. A complete list of spectral features present in the NIR spectra of V2492 Cyg is given in Table 4.

Since V2492 Cyg exhibits CO overtone bandhead emission, we can investigate the characteristics of the $\mathrm{CO}$ emission region using simple numerical models. We follow our previous CO modeling in Aspin et al. (2010) where we studied the bandhead structure in EX Lup during its 2008 extreme eruption. The model we used is based on that of Dent \& Geballe

\footnotetext{
${ }^{8}$ The EX Lup spectrum is from the SpeX spectrograph at the NASA IRTF telescope and has a lower spectral resolution of $\sim 1200$.

${ }^{9}$ The forest of emission lines from 1.15 to $1.22 \mu \mathrm{m}$ are mostly from neutral Fe.
} 
(1991) and Carr \& Tokunaga (1992) and calculates the CO bandhead emission from an isothermal slab of emitting molecular gas characterized by a $\mathrm{CO}$ temperature, $\mathrm{T}_{C O}$, and optical depth in the $\mathrm{CO}$ bandhead, $\tau_{C O}$, and a gas velocity profile. The velocity profiles we have used are either Gaussian of FWHM $v_{g}$ or that of a Keplerian rotating gas disk with velocities defined by the mass of the star, $\mathrm{M}_{*}$, the inner and outer radii of the $\mathrm{CO}$ gas disk, $r_{\text {in }}$ and $r_{\text {out }}$, respectively. Although this model is relatively simple, its ability to produce good matches to CO bandhead emission spectra and its small number of free-parameters lend credence to the uniqueness of the values obtained and the values themselves.

Prior to presenting the results of the CO bandhead modeling, it is informative to comment on the effect of changing the various free parameters used on the model spectrum. This is shown in Figure 14. In panel (a) we plot a model (blue) using the free-parameters $v_{g}=55 \mathrm{~km} \mathrm{~s}^{-1}, \mathrm{~T}_{C O}=3200 \mathrm{~K}$, and $\tau_{C O}=1.8$. In the subsequent panels we vary one of the free-parameters of the model to demonstrate the changes that occur. In panels $(b)$ and $(c$, red) we change $v_{g}$ to 100 and $25 \mathrm{~km} \mathrm{~s}^{-1}$, respectively. Note that the bandhead slope changes as does the visibility of the higher order transitions (resulting in the sinusoidal structure). In panels (d) and (e, green) we change $\mathrm{T}_{C O}$ from 1500 to $4000 \mathrm{~K}$, respectively. The effect of changing $\mathrm{T}_{C O}$ is to raise or lower the level of the emission longward of the bandhead wavelength, specifically, $i$ ) at low $\mathrm{T}_{C O}$ values (e.g. $1500 \mathrm{~K}$ ) the higher order $\mathrm{CO}$ transitions are stronger and hence the bandhead emission remains strong to longer wavelength, and ii) at high $\mathrm{T}_{C O}$ values the opposite is true and the longer wavelength $\mathrm{CO}$ bandhead emission is suppressed. In panels $(f)$ and $\left(g\right.$, purple) we change $\tau_{C O}$ from an optically thin $\tau_{C O}=0.01$ to optically thick $\left(\tau_{C O}=3.6\right)$. This changes the emission strength at wavelengths longward of the bandhead and, in some ways, mimics the effect of changing $\mathrm{T}_{C O}$. If we next consider a disk velocity profile, as shown in panels ( $h$, pink), the so-called 'blue hump 10 appears and the shape of the bandhead changes significantly from that occurring when using a simple Gaussian velocity profile. The disk velocity profile used in panel $\left(h\right.$ ) we had $\mathrm{M}_{*}=0.6 \mathrm{M}_{\odot}$, $\mathrm{T}_{C O}=2500 \mathrm{~K}, \tau_{C O}<0.1$, and $r_{\text {in }}$ and $r_{\text {out }}=1.2$ and $2.0 \times 10^{10} \mathrm{~m}$, respectively. These were the values used for the best-fit model to the EX Lup spectrum shown in Figure 15. If we obscure part of the disk velocity profile, as might be the case in a real star-disk system, we obtain the CO bandhead profiles shown in panels $(i)$ and $(j$, pink). In panel (i) we have removed all blue-shifted velocities from the disk velocity profile and the resultant spectrum shows a significantly red-shifted bandhead peak and peculiar structure in the higher CO transitions. If we remove all red-shifted velocities from the disk velocity profile (panel j) we see a CO bandhead with a 'blue hump' and truncated emission longward of $2.2935 \mu \mathrm{m}$.

\footnotetext{
${ }^{10}$ The 'blue hump' is the name given to the enhanced emission on the blue side of the CO bandhead limit at $2.2935 \mu \mathrm{m})$.
} 
Clearly intermediate obscuration between these two extremes will produce an intermediate CO profile; such spectral peculiarities could be considered as diagnostics of a partly obscured gas disk around a young star.

For V2492 Cyg, we have investigated a wide range of values for the above free parameters for both a Gaussian and disk velocity profile. Specifically, these are $v_{g}=0-150 \mathrm{~km} \mathrm{~s}^{-1}$, $\mathrm{T}_{C O}=1500-4500 \mathrm{~K}, \tau_{C O}=0.01-10, \mathrm{M}_{*}=0.1-10 \mathrm{M}_{\odot}$, and $r_{\text {in }}$ and $r_{\text {out }}=10^{9}-10^{11} \mathrm{~m}$ with $r_{\text {out }}>r_{\text {in }}$. The best-fit model to the observed CO bandhead profile required a Gaussian velocity profile with $v_{g}=55 \pm 10 \mathrm{~km} \mathrm{~s}^{-1}, \mathrm{~T}_{C O}=3200 \pm 200 \mathrm{~K}$, and $\tau_{C O}=1.8 \pm 0.1$. This model (in blue) is shown overlaid on the observed profile (in red) in Figure 15. For comparison, we also show the observed (black) and best-fit model profile (grey) for EX Lup from Aspin et al. (2010). Here, a disk profile was required to fit the observations. Although the spectral resolution of the EX Lup spectrum was considerably higher than that of our Gemini/NIFS spectrum (Keck II/NIRSPEC with R 18,000 as opposed to Gemini/NIFS with R 5,000), a version of the EX Lup spectrum binned to match our V2492 Cyg resolution (green) shows that the observed 'blue-hump' structure, indicative of a disk velocity profile, is still clearly present. We conclude, therefore, that a disk velocity profile is not required to reproduce the observed CO bandhead profile in V2492 Cyg, rather, a simple Gaussian profile is sufficient. This suggests that the CO emission arises from gas with a simple Gaussian velocity profile, perhaps in accretion funnels or in regions with little radial velocity component such as the inner edge of an accretion disk viewed pole-on.

\subsubsection{The UT 2010 November 26/27 NIFS Spectra}

Second epoch NIR NIFS spectra of V2492 Cyg was obtained on UT November 26 (K band) and 27 ( $\mathrm{H}$ band)11. These spectra are presented in Figure 16. As in Figure 13, topleft is the full 1-2.5 $\mu \mathrm{m}$ spectrum of V2492 Cyg together with the same EX Lup spectrum. Bottom-left is a closer view of the H-band spectra and bottom-right is the same for the $\mathrm{K}$ band. Instead of the J-band spectrum at the top-right, we now show a dereddened version of the full 1-2.5 $\mu \mathrm{m}$ spectrum. The dereddening used an extinction of $\mathrm{A}_{V}=20$ mags and shows that such a value for optical extinction produces a spectral slope that more resembles that of EX Lup (which has an intrinsic $A_{V}$ of around 0-1 mags). The EW, line flux, and continuum values for this spectrum are also shown in Table 4 .

The main differences between the UT 2010 October 2/3 and November 26/27 NIR spectra of V2492 Cyg are:

\footnotetext{
${ }^{11}$ No $\mathrm{J}$ band spectrum was acquired due to poor weather conditions and instrument problems.
} 
- The continuum slope is now considerable redder than previously seen. This is consistent with the significant change in NIR colors between the two epochs and related above.

- The water vapor bands exemplified by the 1.7-2.2 $\mu \mathrm{m}$ feature, are more strongly in emission than previously observed.

- The CO overtone bandheads remain in emission although their strength has declined by a factor $\sim 4$ during the intervening period. The profile of the $\mathrm{v}=2-0 \mathrm{CO}$ bandhead at $2.294 \mu \mathrm{m}$ is similar to that seen in October, however, the spectral structure observed is less well defined and the slope of the bandhead itself is slightly shallower. Fitting our CO bandhead emission model to the UT 2010 November 26 data produces a best fit as shown in Figure 17. This model requires $v_{g}=85 \pm 10 \mathrm{~km} \mathrm{~s}^{-1}, \mathrm{~T}_{C O}=4200 \pm 200 \mathrm{~K}$, and $\tau_{C O}=2.0 \pm 0.1$ to reproduce the observations. This represents increases in Gaussian velocity, CO temperature, and optical depth with respect to the values found for the best fit to the UT 2010 October $2 / 3$ data. Since the same instrument and instrumental setup was used for both sets of observations, we consider these changes to be significant at the level determined by the associated uncertainties.

- Molecular hydrogen $\left(\mathrm{H}_{2}\right)$ emission, in the form of the $\mathrm{v}=1-0 \mathrm{~S}(1)$ line at $2.122 \mu \mathrm{m}$, has become more apparent although its intrinsic strength has remained approximately constant at $\sim 1.4 \times 10^{-14}$ ergs $\mathrm{cm}^{-2} \mathrm{~s}^{-1}$. [Fe II] emission at $1.644 \mu \mathrm{m}$ is now detected albeit at a weak level $\left(\sim 3.6 \times 10^{-15}\right.$ ergs $\left.\mathrm{cm}^{-2} \mathrm{~s}^{-1}\right)$. Both these lines are typically associated with the generation of shocks.

- The Br $\gamma$ line flux decreased by a factor $\sim 4$ between UT 2010 October 2/3 and November $26 / 27$. This is very similar to the reduction in both the CO bandhead emission and the $\mathrm{Na}$ I emission at $2.208 \mu \mathrm{m}$. The profile of $\mathrm{Br} \gamma$ is very similar in the two spectra with a (deconvolved) FWHM $200 \mathrm{~km} \mathrm{~s}^{-1}$. The line luminosity in Br $\gamma$ has declined from $0.001 \mathrm{~L}_{\odot}$ on UT 2010 October 2 to $0.0003 \mathrm{~L}_{\odot}$ on UT 2010 November 26, again assuming $\mathrm{A}_{V}=0$ and $\mathrm{d}=600 \mathrm{pc}$.

\subsubsection{A Comparison to the pre-October 2010 NIR Spectra}

Covey et al. (2011) presented NIR spectra of V2492 Cyg taken on UT 2010 July 14 and 18. On these dates the star had an optical brightness of $\mathrm{R}_{P T F} \sim 15.5$ and 16, respectively.

\footnotetext{
${ }^{12}$ PTF stands for the Palomar Transient Factory. Definition of the $\mathrm{R}_{P T F}$ filter is given in Covey et al. (2011).
} 
These values are around 2 and 2.5 mags fainter (on the $\mathrm{R}_{P T F}$ magnitude scale) than at maximum light which occurred in early September 2010. In quiescence (e.g. in July 2009), V2492 Cyg had an $\mathrm{R}_{P T F}$ brightness of $\sim 19$.

We find, in comparison to our subsequent spectra, that:

- On UT 2010 July 14 and 18, V2492 Cyg possessed Br $\gamma$ line fluxes of 1.4 and $2.9 \times 10^{-14} \mathrm{ergs} \mathrm{cm}^{-2} \mathrm{~s}^{-1}$, respectively. This compares to 9 and $2.3 \times 10^{-14} \mathrm{ergs}_{\mathrm{cm}}^{-2} \mathrm{~s}^{-1}$ on UT October 2 and November 26, respectively. If we equate $\operatorname{Br} \gamma$ line flux to accretion then we can conclude that in early October 2010, V2492 Cyg had an accretion rate some $\sim 3-5 \times$ of that in July and November 2010.

- The $\mathrm{v}=1-0 \mathrm{~S}(1) \mathrm{H}_{2}$ emission line at $2.122 \mu \mathrm{m}$ appears to be relatively constant over the period UT 2010 July 14 to November 26. The line flux measured by Covey et al. (2011) and in our spectra was 1, 1.7, 1.3, and $1.5 \times 10^{-14} \mathrm{ergs} \mathrm{cm}^{-2} \mathrm{~s}^{-1}$, on UT 2010 July 14 and 18, September 5, and November 26, respectively. The v=2-1 S(1) $\mathrm{H}_{2}$ line at $2.2477 \mu \mathrm{m}$ line is detected at three of the four epochs, specifically, July 14 and 18, and November 26. Since it is significantly weaker than the $\mathrm{v}=1-0 \mathrm{~S}(1)$ line this is understandable due to the $\mathrm{v}=1-0 \mathrm{~S}(1)$ line flux being the smallest on UT 2010 October 2. Beck et al. (2007) considered the ratio of certain $\mathrm{H}_{2}$ lines for different sources of excitation 13 as a diagnostic for the excitation mechanism in effect. The ratio of the $\mathrm{v}=2-1 \mathrm{~S}(1) / \mathrm{v}=1-0 \mathrm{~S}(1)$ was found to be 0.55, 0.05, and 0.24 for UV pumped fluorescent excitation, continuous C-type shock excitation 14, and jump J-type shock excitation 15 , respectively. On the three dates the $\mathrm{v}=2-1 \mathrm{~S}(1)$ line was detected, the above ratio was $0.24,0.33$, and 0.19 suggesting that the $\mathrm{H}_{2}$ excitation mechanism is likely to be J-type shocks.

- The flux ratio of $\mathrm{Br} \gamma$ emission to Na I $2.206 \mu \mathrm{m}$ emission was found by Lorenzetti et al. (2009) and Antoniucci et al. (2008) to be dependent on the type of young star being observed. Covey et al. (2011), used the ratio of these lines to show that for V2492 Cyg, the value was $\sim 2$ suggesting that the emission in the source was more typical of that

\footnotetext{
${ }^{13}$ Fluorescent excitation ratio calculated by Black \& van Dishoeck (1987). Shock excitation ratios calculated by Smith (1995).

${ }^{14} \mathrm{C}$-shocks occur in regions of strong magnetic field which buffer the higher velocity $\left(\sim 50 \mathrm{~km} \mathrm{~s}{ }^{-1}\right)$ continuous shock from dissociating the $\mathrm{H}_{2}$ molecules (Draine \& McKee 1993).

${ }^{15} \mathrm{~J}$-shocks show a discontinuous change in density, temperature, and velocity across the shock front and are typically of lower velocity $\left(<35 \mathrm{~km} \mathrm{~s}^{-1}\right.$ ) which does not dissociate the $\mathrm{H}_{2}$ molecule (Draine \& McKee $1993)$.
} 
from an EXor/jet driving source. Our two epochs of NIR spectroscopy give a ratio of 2.4 and are hence consistent with the values of Covey et al.

\section{Discussion}

\subsection{Pre-Outburst Spectral Energy Distribution}

Using the photometry in Table 5 we can construct a pre-outburst SED of V2492 Cyg. This is shown graphically in Figure 18. We have used the Robitaille et al. $(2006,2007)$ models and on-line SED fitting too 16 to estimate the contributions to the SED from the stellar photosphere, circumstellar disk, and circumstellar envelope, and to provide insight into their physical nature. As well as specifying the source flux vs. wavelength, the fitting tool also allows one to input a range of distances and interstellar extinctions $\left(\mathrm{A}_{V}\right)$. We have adopted a distance of 500-600 pc and a possible range of interstellar $A_{V}$ of 0-10 mags. The fitting process produces reasonably good fits to the data, however, it is judicious to note that because of the many free parameters, the model may clearly produce a good fit to the data which is not truly representative of the physical nature of the source. Nevertheless, such a procedure does provide insight into the quiescent nature of the source and hence the SED and model fits are considered below.

The SED of V2492 Cyg (Figure 18) rises steeply from the optical to $\sim 3 \mu \mathrm{m}$ where it flattens out to $\sim 100 \mu \mathrm{m}$ then declines into the mm-wave regime. Using the above constraint on distance and interstellar extinction, we find that the fitting procedure produces the bestfit parameters shown in Figure 18 as model \#3009214 (solid line). This model has a mass, effective temperature, and total luminosity consistent with an intermediate-mass Herbig Ae/Be star i.e. $\mathrm{M}_{*}=3.5 \mathrm{M}_{\odot}, \mathrm{T}_{*}=13000 \mathrm{~K}$, and $\mathrm{L}_{t o t}=126 \mathrm{~L}_{\odot}$, respectively. The age of the star is $t_{*} \sim 4 \times 10^{6}$ yrs and it has a total (circumstellar + interstellar) extinction of $\mathrm{A}_{V}=35$ mags. However, almost all of this extinction is unrealistically required to be of interstellar origin.

If we place additional constraints on the best-fit procedure, specifically, $M_{*}<2 M_{*}$, the best-fit model \#3019063 (dashed line) has $\mathrm{M}_{*}=0.7 \mathrm{M}_{\odot}, \mathrm{T}_{*}=3850 \mathrm{~K}, \mathrm{~L}_{t o t}=14 \mathrm{~L}_{\odot}$, and $\mathrm{A}_{V}=12$ mags. However, the source age for this model is small at $t_{*} \sim 2000$ yrs. If we additionally constrain $t_{*}$ to be between $10^{4}$ and $10^{7} \mathrm{yrs}$, the best-fit model \#3015948 (dotted line) has $\mathrm{M}_{*}=1.9 \mathrm{M}_{\odot}, \mathrm{T}_{*}=4350 \mathrm{~K}, \mathrm{~L}_{t o t}=30 \mathrm{~L}_{\odot}$, and $\mathrm{A}_{V}=25$ mags. The age of the young star in this model is $1.5 \times 10^{5}$ yrs. Inspection of Figure 18 shows that models \#3009214 and \#3019063 fit the data reasonably well. However, model \#3015948 under-estimates the flux

\footnotetext{
${ }^{16}$ At URL: http://caravan.astro.wisc.edu/protostars/
} 
from 5-11 $\mu \mathrm{m}$ and over-estimates it from $11-100 \mu \mathrm{m}$. The full list of the above model values are given in Table 6 ,

Taking a somewhat different approach, which is the one adopted here, if constrain the interstellar $\mathrm{A}_{V}$ component to be $<10$ mags (as suggested by the JHK $\mathrm{c}-\mathrm{c}$ diagram) and average the top 20 best-fit models that satisfy this constraint, we obtain the final column of values in Table 6. As can be seen, the top 20 models show free-parameters with, in most cases, reasonable $1 \sigma$ standard deviations. These values are most likely the best we can obtain for V2492 Cyg. The source age is again very small at $2.1 \pm 0.2 \times 10^{3}$ yrs, however, it is calculated from the derived values of stellar radius, temperature, and mass and so are model dependent (B. Whitney 2011, private communication). All we can likely say is that the top $20 \mathrm{~A}_{V}$-constrained models indicate that V2492 Cyg is relatively young.

\subsection{Accretion Luminosities and Rates}

It is possible to investigate the accretion luminosity, $\mathrm{L}_{a c c}$, and mass accretion rate, $\dot{\mathrm{M}}$, using the V-band flux excess, the $\mathrm{H} \alpha$ FW10\% values, and the observed $\mathrm{Pa} \beta$ and $\mathrm{Br} \gamma$ fluxes as we have previously attempted for V1647 Ori (Aspin, Beck \& Reipurth 2008) and EX Lup (Aspin et al. 2010). However, the value of $\mathrm{A}_{V}$ to adopt in the post-outburst period is critically important to the results obtained. In the JHK c-c diagram (Figure 6a), the location of V2492 Cyg on UT 2010 September 5 (point \#4) was close to the upper end of the CTTS locus. This suggests that soon after the outburst occurred, the source was not particularly reddened by overlying extinction. If we deredden its location to a slightly extended CTTS locus (point B), we find $\mathrm{A}_{V} \sim 2$ mag. This is in contrast to its location in 2006 when it was both highly reddened and possessed a significant K-band thermal excess. On UT 2010 November 25 (point \#8), it appears that the overlying extinction had increased with respect to that on October 2. Dereddening to the CTTS locus gives an $\mathrm{A}_{V} \sim 7$ mags. Since the $1.282 \mu \mathrm{m} \mathrm{Pa} \beta$ flux is significantly more affected by extinction than the $2.166 \mu \mathrm{m}$ $\operatorname{Br} \gamma$ flux, we only use the fluxes from the longer wavelength line below.

Above we noted that the observed $\operatorname{Br} \gamma$ line fluxes were 9 and $2.3 \times 10^{-14} \mathrm{ergs} \mathrm{cm}^{-2} \mathrm{~s}^{-1}$ on UT 2010 October 2 and November 26, respectively. Dereddening these values by $A_{V}=2$ and 7 mags, respectively, gives fluxes of $1.1 \times 10^{-13}$ and $4.7 \times 10^{-14} \mathrm{ergs} \mathrm{cm}^{-2} \mathrm{~s}^{-1} \mathrm{~s}^{-1}$. Following Muzerolle et al. (1998) and Gullbring et al. (1998), we can derive $\mathrm{L}_{a c c}$ and $\dot{\mathrm{M}}$, however, for this determination we have to adopt values for the parameters $\mathrm{M}_{*}$ (the mass of the young star), $\mathrm{L}_{*}$ (the stellar luminosity), $\mathrm{T}_{*}$ (the star's effective temperature), and $r_{i n}$ (the inner radius of the accretion disk). As we have seen, the SED fitting gave ambiguous results. However, if we adopt the most likely physical nature for V2492 Cyg, that of a young, low- 
mass $\mathrm{T}$ Tauri star, we can constrain the above parameters to be $\mathrm{M}_{*} \sim 0.7 \mathrm{M}_{\odot}, \mathrm{T}_{*} \sim 4000 \mathrm{~K}$, $\mathrm{R}_{*} \sim 5-6 \mathrm{R}_{\odot}$ and using the standard luminosity, temperature and stellar radius relationship, $\mathrm{L}_{*} \sim 7 \mathrm{~L}_{\odot}$. Gullbring et al. (1998) adopted $r_{i n} \sim 5 \mathrm{R}_{*}$ as a typical value for CTTSs and we also adopt this value. For the UT 2010 October $2 \mathrm{Br} \gamma$ flux and an $\mathrm{A}_{V}=2$, we derive $\mathrm{L}_{a c c}=5.9 \pm 2 \mathrm{~L} \odot$ and $\dot{\mathrm{M}}=1.8 \pm 0.4 \times 10^{-6} \mathrm{M}_{\odot} \mathrm{yr}^{-1}$. For the UT 2010 November $26 \mathrm{Br} \gamma$ flux and an $\mathrm{A}_{V}=7$, these values become $\mathrm{L}_{a c c}=2.0 \pm 0.7 \mathrm{~L}_{\odot}$ and $\dot{\mathrm{M}}=6.3 \pm 0.5 \times 10^{-7} \mathrm{M}_{\odot} \mathrm{yr}^{-1}$. If we perform the same analysis with the $\operatorname{Br} \gamma$ fluxes from Covey et al. (2011) we obtain $\mathrm{L}_{a c c}$ values of $2.1 \pm 0.5$ and $5.2 \pm 1.5 \mathrm{~L}_{\odot}$ for UT July 14 and July 18, respectively (using $\mathrm{A}_{V}=12$ mags on both dates). Using the same stellar/disk parameters, these convert to $\dot{\mathrm{M}}$ rates of $6.4 \pm 1.5 \times 10^{-7}$ and $1.6 \pm 0.5 \times 10^{-6} \mathrm{M}_{\odot} \mathrm{yr}^{-1}$. The above results are shown in tabular form in Table 7. Although the derived values are dependent on the assumed star/disk parameters, using the same values for all four epochs allows us to inter-compare our results. We find that the accretion luminosity and mass accretion rate increases by a factor 2.5 between UT 2010 July 14 and 18, increased still further by a factor 1.1 between UT July 18 and October 2, then declined by a factor 2.9 between UT 2010 October 2 and November 26. If we integrate the accretion rate over the period UT 2010 July 14 to November 26 we obtain a total mass accreted of $\sim 2 \times 10^{-4} \mathrm{M}_{\odot}$.

Figure 9 shows that V2492 Cyg has a deconvolved $\mathrm{H} \alpha \mathrm{FW} 10 \%$ of $~ 550 \mathrm{~km} \mathrm{~s}^{-1}$. Using equation (1) of Natta et al. (2004) we obtain a value for $\dot{M}$ of $\sim 3 \times 10^{-8} \mathrm{M}_{\odot} \mathrm{yr}^{-1}$. The uncertainties on this value (from Natta et al. equation 1 ) give a possible range of rates of $6 \times 10^{-9}$ to $1 \times 10^{-7} \mathrm{M}_{\odot} \mathrm{yr}^{-1}$. Even the high end of this range is $10 \times$ smaller than the value above derived using the dereddened $\operatorname{Br} \gamma$ flux.

Despite the significant uncertainties of the above analysis, we can conclude that the above estimates of $\dot{M}$ for V2492 Cyg during outburst are considerably lower than the expected values in a FUor eruption e.g. $10^{-5}$ to $10^{-4} \mathrm{M}_{\odot} \mathrm{yr}^{-1}$. We find, therefore, that the outburst of V2492 Cyg seems more consistent with those observed in EXors than in FUors.

\subsection{Indicators of Outflow and Stellar/Disk Winds}

As discussed by Covey et al. (2011), Hartigan, Edwards, \& Ghandour (1995) presented a relationship between mass-loss rate and the luminosity of certain forbidden optical emission lines related to shock-excitation by outflowing gas. Using their equations A8 and A10, Covey et al. estimated the mass outflow rate, $\dot{\mathrm{M}}_{\text {wind }}$, to be $7 \times 10^{-7}$ and $2 \times 10^{-6} \mathrm{M}_{\odot} \mathrm{yr}^{-1}$ from the $6716 \AA[\mathrm{S} \mathrm{II}]$ line and the $6300 \AA[\mathrm{O}$ I] line, respectively. Stressing the large uncertainties in these values due to the assumed values for visual extinction and the physical properties of the shock region, they nonetheless showed that significant mass outflow is occurring during 
periods of intense mass accretion. Using both the [O I] line fluxes from Covey et al. (2011) and or own from Table3, we have re-calculated mass-loss rates using a standard set of input parameters. The only quantity we have varied between epochs is the overlying visual extinction. This we estimated from the JHK c-c diagram shown in Figure6a. Table 8 shows the estimated mass loss-rates for the four epochs for which [O I] emission line fluxes are available. We only use the [O I] line here since it is stronger than the [S II] lines present and, hence, the fluxes are considered more reliable. For all calculations, we assume values for the electron density, $\mathrm{N}_{e}$, equal to the $[\mathrm{O} \mathrm{I}]$ critical density, $\mathrm{N}_{c}=1.97 \times 10^{6} \mathrm{~cm}^{-3}$, the projected size of the aperture on the plane of the sky, $1_{\text {perp }}=2.62 \times 10^{15} \mathrm{~cm}$ (corresponding to $0^{\prime \prime} .3$ at $600 \mathrm{pc}$ ), and the wind velocity component in the plane of the sky, $\mathrm{v}_{\text {perp }}=150 \mathrm{~km} \mathrm{~s}^{-1}$. We note that $\dot{\mathrm{M}}_{\text {wind }}$ scales as $\left(1+\mathrm{N}_{c} / \mathrm{N}_{e}\right), \mathrm{l}_{\text {perp }}^{-1}$, and $\mathrm{v}_{\text {perp }}^{-1}$. For the observed [O I] fluxes and assumed values of $\mathrm{A}_{V}$, we estimate mass-loss rates of $1-2 \times 10^{-6} \mathrm{M}_{\odot} \mathrm{yr}^{-1}$ in July 2010 dropping very significantly to $2-3 \times 10^{-9} \mathrm{M}_{\odot} \mathrm{yr}^{-1}$ in September 2010 , and increasing to $\sim 4 \times 10^{-8} \mathrm{M}_{\odot} \mathrm{yr}^{-1}$ in November 2010. Clearly, these values are highly uncertain and evidently dependent on the assumed $\mathrm{A}_{V}$ value. However, the overall trend indicates that the mass-loss rate in the wind was highest in July $2010\left(\mathrm{R}_{P T F}=15.7\right)$, prior to the September peak in optical brightness, then declined by three orders of magnitude at the time of the September 2010 brightness peak $\left(\mathrm{R}_{P T F}=13.6\right)$, and then increased by a factor $\sim 10$ as the source faded back to a more quiescent-like optical brightness in November $2010\left(\mathrm{R}_{P T F}=18.0\right)$. This behavior is somewhat peculiar since the expectation would more likely be that the mass-loss rate would increase significantly immediately after the dramatic increase in brightness occurred.

\section{Summary}

What we have learned about the young star V2492 Cyg is summarized below.

1. The outburst of V2492 Cyg appears to be more representative of those occurring in EXor rather than FUor variables. Its optical and NIR emission line spectra and shorter timescale brightness variations are much more like those found in EX Lup and VY Tau than in any of the classical FUors or FUor-like objects.

2. Based on Spitzer c-c diagrams, V2492 Cyg is designated a Class I protostar consistent with the evolutionary state determined by Covey et al. (2011).

3. Although it is not clear when precisely the outburst in V2492 Cyg began, we can obtain a timeline, albeit incomplete, of events by utilizing the light-curve of Covey et al. (2011) together with our Figure 5. In quiescence, V2492 Cyg appears to be optically faint with an R-band magnitude of 18-19. The source had such a brightness in the 
USNO-B1 survey from 1979.9 and in September and October 2009 (Covey et al. 2011). However, rather than being relatively constant in optical brightness in quiescence, some small-scale variability seems to be present (hence the above quiescent magnitude range). For example, Itagaki (2010) presented photometry from UT 2009 May 7 which showed V2492 Cyg had an unfiltered optical magnitude of 15.4. This corresponds to R 15.8 (see Figure 5 for a comparison of unfiltered to R-band photometry). At some time between UT 2009 October 25 (the last 2009 point from Covey et al. (2011) and UT 2009 December 19 (the first of the Itagaki 2010 points shown in Figure 5) the current activity period began.

4. A significant fraction of the total change in optical and NIR brightness is likely due to the disappearance and re-formation of dust along our line-of-sight. Although the changes in NIR colors do not correspond well to those expected from previously used extinction laws, the more or less linear (in $\log _{10}$-space) change in location of V2492 Cyg in a JHK c-c diagram strongly suggests that extinction variations are the dominant factor. Dust sublimation and re-formation have been previously observed in the eruptive variable V1647 Ori; the dust is sublimated by an accretion burst and then re-forms as the accretion process returns to a more stable state.

5. During the outburst, V2492 Cyg developed a strong stellar/disk wind seen as blueshifted absorption on several strong emission lines. For example, on UT 2010 September 5 both $\mathrm{H} \alpha$ and the far-red Ca II triplet lines showed velocities of $\sim-200 \mathrm{~km} \mathrm{~s}^{-1}$ in the sub-continuum blue-shifted absorption minimum. Between that date and UT 2010 November 26, the velocity of the minimum absorption trough on two of the Ca II triplet lines reduced to $\sim 125 \mathrm{~km} \mathrm{~s}^{-1}$ (this trough disappeared on the other Ca II line) while the sub-continuum absorption in $\mathrm{H} \alpha$ became a low-velocity absorption on the blue-wing of the emission profile. An emission line typically associated with mass-loss is the $6300 \AA[\mathrm{O}$ I] line. The dereddened [O I] line fluxes suggest that the star/disk wind was strongest prior to the September 2010 brightness peak. At the time of the September 2010 maximum, it had declined by three orders of magnitude but then increased in strength by a factor 10 some 3 months later.

6. The NIR CO overtone bandhead emission from UT 2010 October 2 indicates that the emitting region has a temperature, $\mathrm{T}_{C O} \sim 3200 \mathrm{~K}$, is somewhat optically thick, and has a Gaussian velocity dispersion, $\mathrm{v}_{g} \sim 55 \mathrm{~km} \mathrm{~s}^{-1}$. Approximately two months later, both $\mathrm{T}_{C O}$ and $\mathrm{v}_{g}$ had increased to $\sim 4200 \mathrm{~K}$ and $85 \mathrm{~km} \mathrm{~s}^{-1}$, respectively. This is consistent with the buildup of gaseous, circumstellar material closer to the star as the eruption subsides. The lack of evidence for this gaseous material being in the form of a circumstellar disk either suggests that the emitting region is located elsewhere, 
perhaps in accretion funnels, or that the disk is viewed either relatively face-on or partly obscured from directly view. For a $0.7 \mathrm{M}_{\odot}$ central star with a Keplerian disk, velocities of 55 and $85 \mathrm{~km} \mathrm{~s}^{-1}$ correspond orbital radii of 0.2 and $0.09 \mathrm{AU}$, respectively.

7. Optical (e.g. $[\mathrm{S} \mathrm{II}]$ ) and NIR (e.g. $\mathrm{H}_{2}$ ) shock-excited emission lines were present in the earliest Covey et al. (2011) spectrum from UT 2010 July 14, absent (or very weak) in our UT 2010 September 5 spectrum, and present again in our most recent spectrum from UT 2010 November 25. There is no evidence as to the origin of these lines but possibilities include shocks associated with the accretion flow or those created by outflowing gas such as in a collimated Herbig-Haro flow. We cannot distinguish between these mechanisms at this time.

8. The pre-outburst SED of V2492 Cyg has been fitted using the model SEDs of Robitaille et al. (2006, 2007). The best fits indicate that the star is at a very young evolutionary stage and is of low-mass with $\mathrm{M}_{*} \sim 0.5 \mathrm{M}_{\odot}, \mathrm{T}_{*} \sim 3600 \mathrm{~K}, \mathrm{~L}_{\text {tot }} \sim 20 \mathrm{~L}_{\odot}$, and $\mathrm{R}_{*} \sim 7 \mathrm{R}_{\odot}$. Due to its age, the star still possesses a significant circumstellar envelope $\left(\mathrm{M}_{e n v} \sim 0.02 \mathrm{M}_{\odot}\right)$ and disk $\left(\mathrm{M}_{d i s k} \sim 0.01 \mathrm{M}_{\odot}\right)$. All indications are that it is still in the Class I protostar phase.

9. From an analysis of the $\operatorname{Br} \gamma$ line fluxes from Covey et al. (2011) and from our spectra, we find that the accretion luminosity and rate fall in the ranges $\mathrm{L}_{a c c}=1-6 \mathrm{~L}_{\odot}$ and $\dot{\mathrm{M}}=0.4-6 \times 10^{-6} \mathrm{M}_{\odot} \mathrm{yr}^{-1}$, respectively. Although these values depend on the assumed $\mathrm{A}_{V}$ at the time of observations, we can say with reasonable confidence that these accretion parameter values are significantly lower than found in FUors/FUor-like objects and more like those occurring in EXor outbursts.

It remains to be seen how V2492 Cyg evolves and whether it continues to exhibit shorttimescale variability or returns to a more quiescent state. Nevertheless, our conclusion is that it appears to be another example of an EXor variable similar in nature to EX Lup although apparently significantly younger. Continuous optical monitoring of the star by the AAVSO observers will clearly greatly assist in a better understanding of this enigmatic young stellar object.

Acknowledgments We are extremely grateful to the referee, Barbara Whitney, for many useful comments on the manuscript, in particular, on the interpretation of the best-fit SED models. We wish to express our sincere thanks to Nancy Levinson for allocating the Director's time used to obtain our GMOS-N, NIRI, and NIFS Gemini-North observations. We are grateful to the AAVSO and their dedicated observers who continue to provide high quality optical photometry of V2492 Cyg. We are pleased to acknowledge Kevin Covey for 
valuable discussions and providing some additional information on his recent paper and on the source itself. We thanks Bo Reipurth for useful discussions and critical reading of the

manuscript. This project was supported by the Gemini Observatory, which is operated by the Association of Universities for Research in Astronomy, Inc., on behalf of the international Gemini partnership of Argentina, Australia, Brazil, Canada, Chile, the UK, and the US. This work is based in part on data obtained as part of the UKIRT Infrared Deep Sky Survey. This publication makes use of data products from the Two Micron All Sky Survey, which is a joint project of the University of Massachusetts and the Infrared Processing and Analysis Center/California Institute of Technology, funded by the National Aeronautics and Space Administration and the National Science Foundation. This work is based in part on observations made with the Spitzer Space Telescope, which is operated by the Jet Propulsion Laboratory, California Institute of Technology under a contract with NASA. Support for this work was provided by NASA through an award issued by JPL/Caltech. This research is based in part on observations with AKARI, a JAXA project with the participation of ESA.

\section{REFERENCES}

Allen, L.E. et al. (2002) ApJ, 566, 993

Ambartsumian, V.A. 1971, Astrophysics, 7, 331

Aspin, C., Reipurth, B., Herczeg, G.J. \& Capak, P., 2010, ApJ, 719, 50

Aspin, C., Beck, T.L., \& Reipurth, B. 2008, AJ, 135, 423

Aspin, C., \& Reipurth, B. 2009, AJ, 138, 1137

Bally, J. \& Reipurth, B. 2003, AJ, 126, 893

Briceno, C. et al. 2004, ApJ, 606, L123

Covey, K. et al. 2011, in press

Davies, R.L. et al. 1997, SPIE, 2871, 1099

Dobashi, K. et al. 2005, PASJ, 57, S1

Draine, B.T. \& McKee, C.F. 1993, ARA\&A, 31, 373

Greene, T.P. \& Meyer, P. 1995, ApJ, 450, 233

Gutermuth, R.A., Megeath, S.T., Muzerolle, J., et al. 2004, ApJS, 154, 374 
Gutermuth, R.A., Myers, P.C., Megeath, S.T., et al. 2008, ApJ, 674, 336

Hartmann, L., \& Kenyon, S.J. 1985, ApJ, 299, 462

Hartmann, L. \& Kenyon, S.J. 1996, ARAA, 34, 207

Herbig, G.H. 1966, Vistas in Astronomy, 8, 109

Herbig, G.H. 1977, ApJ, 217, 693

Herbig, G.H. 1989, in ESO Workshop on Low Mass Star Formation and Pre-Main Sequence Objects, ed. B. Reipurth, p. 233

Herbig, G.H. 1990, ApJ, 360, 639

Herbig, G.H. 2008, AJ, 135, 637

Herbig, G.H. \& Harlan, E.A. 1971, IBVS, 543

Herbig, G.H., Petrov, P.P., \& Duemmler, R. 2003, ApJ, 595, 384

Hodapp, K.-W. et al. 2003, PASP, 115, 1388

Hook, I., Jorgensen, I., Allington-Smith, J.R., Davies, R.L., Metcalfe, N., Murowinski, R.G., \& Crampton, D. 2004, PASP, 116, 425

Itagaki, K. 2010, CBET, 2426

Laugalys, V. et al. 2006, Baltic Astron., 15, 483

Lawrence, A., et al. 2007, MNRAS, 379, 1599

Meinunger, L. 1969, Mitt. über Veränderlichen Sterne, 5, 47

McGregor, P.J. et al. 2003, SPIE, 4841.1581

Murakami, H., Baba, H., \& Barthel, P., et al. 2007, PASJ, 59, 369

Osorio, M., D’Alessio, P., Muzerolle, J., Calvet, N., \& Hartmann, L. 2003, RevMexAA (Serie de Conferencias) 15, 142

Petrov, P.P. \& Herbig, G.H. 1992, ApJ, 392, 209

Petrov, P.P. \& Herbig, G.H. 2008, AJ, 136, 676 
Reipurth, B. \& Schneider, N. 2008, in Handbook of Star Forming Regions Vol. I, ed. B. Reipurth, ASP, p. 36

Reipurth, B. \& Aspin, C. 2004, ApJ, 606, L119

Robitaille, T.P., Whitney, B.A., Indebetouw, R., Wood, K., \& Denzmore, P. 2006, ApJS, 167,256

Robitaille, T.P., Whitney, B.A., Indebetouw, R., \& Wood, K. 2007, ApJS, 169, 328

Semkov, E. \& Peneva, S. 2010a, ATel, 2801

Semkov, E. \& Peneva, S. 2010b, ATel, 2819

Skrutskie, M.F. et al. 2006, AJ, 131, 1163

Wachmann, A.A. 1954, Zs. f. Ap. 35, 74

Welin, G. 1971, A\&A, 12, 312

Whitney, B.A., Kenyon, S.J., \& Gomez, M. 1997, ApJ, 485, 703 
Table 1. Observation Log

\begin{tabular}{|c|c|c|c|}
\hline UT Date & JD & $\begin{array}{l}\text { Telescope/ } \\
\text { Instrument }\end{array}$ & Details \\
\hline 2010 Sep 5 & 2455445 & Gemini-N/GMOS & r' imaging \\
\hline 2010 Sep 5 & 2455445 & Gemini-N/GMOS & B600 spectroscopy \\
\hline 2010 Sep 5 & 2455445 & Gemini-N/NIRI & $\mathrm{J}, \mathrm{H}, \mathrm{K}^{\prime}, \mathrm{L}^{\prime}$ photometry \\
\hline 2010 Oct 2 & 2455472 & Gemini-N/NIFS & K-band spectroscopy \\
\hline 2010 Oct 3 & 2455473 & Gemini-N/NIFS & $\mathrm{J}, \mathrm{H}$ spectroscopy \\
\hline 2010 Nov 25 & 2455525 & Gemini-N/GMOS & B600 spectroscopy \\
\hline 2010 Nov 25 & 2455525 & Gemini-N/NIRI & $\mathrm{J}, \mathrm{H}, \mathrm{K}^{\prime}, \mathrm{L}^{\prime}$ photometry \\
\hline 2010 Nov 26 & 2455526 & Gemini-N/NIFS & K-band spectroscopy \\
\hline 2010 Nov 27 & 2455527 & Gemini-N/NIFS & H-band spectroscopy \\
\hline
\end{tabular}


Table 2. NIR Photometry

\begin{tabular}{lcccr}
\hline \hline Filter & $\begin{array}{c}\text { 2000/06/10 } \\
(2 \mathrm{MASS})\end{array}$ & $\begin{array}{c}2006 / 06 / 10 \\
\text { (UKIDSS })\end{array}$ & $\begin{array}{c}2010 / 09 / 05 \\
(\text { Gemini })\end{array}$ & $\begin{array}{c}2010 / 11 / 25 \\
(\text { Gemini })\end{array}$ \\
\hline $\mathrm{J}$ & $>15.8$ & $19.40 \pm 0.09$ & $10.58 \pm 0.1$ & $13.13 \pm 0.1$ \\
$\mathrm{H}$, & $>15.1$ & $16.55 \pm 0.02$ & $9.33 \pm 0.1$ & $11.45 \pm 0.1$ \\
$\mathrm{~K}$ & $>14.3$ & $13.15 \pm 0.01$ & $8.12 \pm 0.1$ & $9.63 \pm 0.1$ \\
$\mathrm{~L} / \mathrm{L}$, & - & - & $5.36 \pm 0.1$ & $6.05 \pm 0.1$ \\
$\mathrm{~J}-\mathrm{H}$ & - & $2.85 \pm 0.09$ & $1.25 \pm 0.14$ & $1.68 \pm 0.14$ \\
$\mathrm{H}-\mathrm{K}{ }^{\prime}$ & - & $3.40 \pm 0.02$ & $1.21 \pm 0.14$ & $1.82 \pm 0.14$ \\
$\mathrm{~K}^{\prime}-\mathrm{L}$, & - & - & $2.76 \pm 0.14$ & $3.58 \pm 0.14$ \\
\hline
\end{tabular}


Table 3. Optical Spectral Features with $\mathrm{EW} \geq 1 \AA$ on One of the Two Observation Dates

\begin{tabular}{|c|c|c|c|c|c|c|c|}
\hline Line & $\begin{array}{c}\lambda \\
(\AA)\end{array}$ & $\begin{array}{l}\mathrm{EW}^{\mathrm{a}} \\
(\AA)\end{array}$ & $\begin{array}{c}\text { Line Flux } \\
\left(\operatorname{ergs~} \mathrm{cm}^{-2} \mathrm{~s}^{-1}\right) \\
05 \text { Septen }\end{array}$ & $\begin{array}{l}\quad \text { Continuum } \\
\left(\operatorname{ergs~cm}{ }^{-2} \mathrm{~s}^{-1} \AA^{-1}\right) \\
\text { ber } 2010\end{array}$ & $\begin{array}{c}\mathrm{EW}^{\mathrm{a}} \\
(\AA)\end{array}$ & $\begin{array}{c}\text { Line Flux } \\
\left(\text { ergs } \mathrm{cm}^{-2} \mathrm{~s}^{-1}\right) \\
25 \text { Novem }\end{array}$ & $\begin{array}{l}\quad \text { Continuum } \\
\left(\operatorname{ergs~} \mathrm{cm}^{-2} \mathrm{~s}^{-1} \AA^{-1}\right) \\
\text { er } 2010\end{array}$ \\
\hline Fe I & 6136 & -1.5 & $1.53(-14)^{\mathrm{b}}$ & $1.02(-14)$ & -2.4 & $2.64(-16)$ & $1.10(-16)$ \\
\hline Fe II & 6148 & -1.0 & $1.03(-14)$ & $1.03(-14)$ & -0.9 & $1.01(-16)$ & $1.12(-16)$ \\
\hline Fe I & 6192 & -0.8 & $8.53(-15)$ & $1.07(-14)$ & -1.0 & $1.19(-16)$ & $1.19(-16)$ \\
\hline Fe II & 6238 & -0.9 & $9.84(-15)$ & $1.09(-14)$ & -1.6 & $1.99(-16)$ & $1.24(-16)$ \\
\hline $\mathrm{Fe}$ II & 6247 & -0.8 & $8.78(-15)$ & $1.10(-14)$ & -1.1 & $1.37(-16)$ & $1.25(-16)$ \\
\hline$[\mathrm{O} \mathrm{I}]$ & 6300 & -1.3 & $1.44(-14)$ & $1.11(-14)$ & -38.9 & $4.94(-15)$ & $1.27(-16)$ \\
\hline$[\mathrm{O}$ I $]$ & 6364 & -0.6 & $6.77(-15)$ & $1.13(-14)$ & -13.3 & $1.72(-15)$ & $1.30(-16)$ \\
\hline Fe I & 6393 & -0.7 & $8.02(-15)$ & $1.15(-14)$ & -1.7 & $2.24(-16)$ & $1.32(-16)$ \\
\hline Fe I & 6399 & -0.7 & $8.05(-15)$ & $1.15(-14)$ & -1.5 & $1.99(-16)$ & $1.33(-16)$ \\
\hline Fe II & 6433 & -2.3 & $2.73(-14)$ & $1.19(-14)$ & -3.1 & $4.27(-16)$ & $1.38(-16)$ \\
\hline $\mathrm{Ca} \mathrm{II} / \mathrm{Fe} \mathrm{II}^{\mathrm{c}}$ & 6456 & -0.8 & $9.74(-15)$ & $1.22(-14)$ & -1.5 & $2.14(-16)$ & $1.43(-16)$ \\
\hline Fe I & 6496 & -0.9 & $1.16(-14)$ & $1.28(-14)$ & -2.4 & $3.66(-16)$ & $1.52(-16)$ \\
\hline Fe II & 6516 & -2.6 & $3.43(-14)$ & $1.32(-14)$ & -3.6 & $5.68(-16)$ & $1.58(-16)$ \\
\hline$[\mathrm{N}$ II $]$ & 6548 & -0.7 & $9.61(-15)$ & $1.37(-14)$ & -1.3 & $2.16(-16)$ & $1.66(-16)$ \\
\hline $\mathrm{H} \alpha$ & 6563 & -16.3 & $2.28(-13)$ & $1.40(-14)$ & -27.6 & $4.68(-15)$ & $1.70(-16)$ \\
\hline$[\mathrm{N}$ II $]$ & 6592 & -1.2 & $1.72(-14)$ & $1.44(-14)$ & -2.0 & $3.52(-16)$ & $1.76(-16)$ \\
\hline Fe I & 6664 & $\ldots{ }^{d}$ & $\ldots$ & $\ldots$ & -1.0 & $1.84(-16)$ & $1.84(-16)$ \\
\hline Fe I & 6677 & -0.8 & $1.19(-14)$ & $1.48(-14)$ & -1.3 & $2.39(-16)$ & $1.84(-16)$ \\
\hline$[\mathrm{S}$ II $]$ & 6716 & $\ldots$ & $\ldots$ & $\ldots$ & -2.8 & $5.15(-16)$ & $1.84(-16)$ \\
\hline$[\mathrm{S}$ II $]$ & 6731 & $\ldots$ & $\ldots$ & $\ldots$ & -5.1 & $9.37(-16)$ & $1.84(-16)$ \\
\hline$[\mathrm{Fe} \mathrm{II}]$ & 7155 & $\ldots$ & $\ldots$ & $\ldots$ & -8.4 & $2.40(-15)$ & $2.86(-16)$ \\
\hline
\end{tabular}


Table 3-Continued

\begin{tabular}{|c|c|c|c|c|c|c|c|}
\hline Line & $\begin{array}{c}\lambda \\
(\AA)\end{array}$ & $\begin{array}{l}\mathrm{EW}^{\mathrm{a}} \\
(\AA)\end{array}$ & $\begin{array}{c}\text { Line Flux } \\
\left(\text { ergs } \mathrm{cm}^{-2} \mathrm{~s}^{-1}\right) \\
05 \text { Septen }\end{array}$ & $\begin{array}{l}\quad \text { Continuum } \\
\left(\text { ergs } \mathrm{cm}^{-2} \mathrm{~s}^{-1} \AA^{-1}\right) \\
\text { er } 2010\end{array}$ & $\begin{array}{l}\mathrm{EW}^{\mathrm{a}} \\
(\AA)\end{array}$ & $\begin{array}{c}\text { Line Flux } \\
\left(\operatorname{ergs~cm}^{-2} \mathrm{~s}^{-1}\right) \\
25 \text { Novem }\end{array}$ & $\begin{array}{l}\quad \text { Continuum } \\
\left(\text { ergs } \mathrm{cm}^{-2} \mathrm{~s}^{-1} \AA^{-1}\right) \\
\text { er } 2010\end{array}$ \\
\hline Fe II & 7169 & $\ldots$ & $\ldots$ & $\ldots$ & -2.2 & $6.39(-16)$ & $2.90(-16)$ \\
\hline$[\mathrm{Ca} I \mathrm{II}]$ & 7291 & -1.5 & $2.90(-14)$ & $1.93(-14)$ & -2.2 & $6.75(-16)$ & $3.07(-16)$ \\
\hline Fe II & 7308 & -0.6 & $1.15(-14)$ & $1.91(-14)$ & -1.0 & $3.06(-16)$ & $3.06(-16)$ \\
\hline [Ca II] & 7324 & -0.9 & $1.70(-14)$ & $1.89(-14)$ & -2.2 & $6.73(-16)$ & $3.06(-16)$ \\
\hline $\mathrm{Na} I ?^{\mathrm{e}}$ & 7374 & $\ldots$ & $\ldots$ & $\ldots$ & -2.8 & $8.47(-16)$ & $3.03(-16)$ \\
\hline$[\mathrm{Fe} \mathrm{II}]$ & 7388 & -0.8 & $1.47(-14)$ & $1.84(-14)$ & -2.3 & $6.94(-16)$ & $3.02(-16)$ \\
\hline Si I? ${ }^{e}$ & 7409 & $\ldots$ & $\ldots$ & $\ldots$ & -1.3 & $3.91(-16)$ & $3.01(-16)$ \\
\hline Fe II & 7448 & -0.6 & $1.11(-14)$ & $1.85(-14)$ & -3.6 & $1.08(-15)$ & $3.00(-16)$ \\
\hline Fe II & 7461 & -1.2 & $2.23(-14)$ & $1.86(-14)$ & -1.2 & $3.61(-16)$ & $3.01(-16)$ \\
\hline Fe I & 7618 & $\ldots$ & $\ldots$ & $\ldots$ & -4.1 & $1.41(-15)$ & $3.43(-16)$ \\
\hline K I & 7665 & +3.8 & $8.58(-14)$ & $2.26(-14)$ & +4.3 & $1.59(-15)$ & $3.70(-16)$ \\
\hline K I & 7699 & +2.7 & $6.33(-14)$ & $2.34(-14)$ & +3.2 & $1.25(-15)$ & $3.91(-16)$ \\
\hline Fe II & 7711 & -1.9 & $4.51(-14)$ & $2.37(-14)$ & -2.4 & $9.56(-16)$ & $3.98(-16)$ \\
\hline $\mathrm{O} \mathrm{I}^{\mathrm{f}}$ & 7774 & +3.3 & $8.26(-14)$ & $2.50(-14)$ & +1.9 & $8.17(-16)$ & $4.30(-16)$ \\
\hline Fe I & 7998 & $\ldots$ & $\ldots$ & $\ldots$ & -1.1 & $4.83(-16)$ & $4.39(-16)$ \\
\hline $\mathrm{Fe} I$ & 8046 & -0.9 & $2.20(-14)$ & $2.45(-14)$ & -1.2 & $5.13(-16)$ & $4.28(-16)$ \\
\hline Fe I & 8219 & -0.6 & $1.50(-14)$ & $2.50(-14)$ & -1.1 & $4.93(-16)$ & $4.48(-16)$ \\
\hline Fe I & 8327 & -1.4 & $3.97(-14)$ & $2.84(-14)$ & -2.9 & $1.52(-15)$ & $5.23(-16)$ \\
\hline H I (Pa 24) & 8334 & $\ldots$ & $\ldots$ & $\ldots$ & -1.1 & $5.81(-16)$ & $5.28(-16)$ \\
\hline Fe I & 8388 & -2.7 & $8.19(-14)$ & $3.03(-14)$ & -4.3 & $2.43(-15)$ & $5.66(-16)$ \\
\hline H I (Pa 18) & 8435 & -0.8 & $2.53(-14)$ & $3.16(-14)$ & -1.5 & $8.93(-16)$ & $5.96(-16)$ \\
\hline
\end{tabular}


Table 3-Continued

\begin{tabular}{|c|c|c|c|c|c|c|c|}
\hline Line & $\begin{array}{c}\lambda \\
(\AA)\end{array}$ & $\begin{array}{l}\mathrm{EW}^{\mathrm{a}} \\
(\AA)\end{array}$ & $\begin{array}{c}\text { Line Flux } \\
\left(\text { ergs } \mathrm{cm}^{-2} \mathrm{~s}^{-1}\right)\end{array}$ & $\begin{array}{c}\text { Continuum } \\
\left(\operatorname{ergs~} \mathrm{cm}^{-2} \mathrm{~s}^{-1} \AA^{-1}\right)\end{array}$ & $\begin{array}{l}\mathrm{EW}^{\mathrm{a}} \\
(\AA)\end{array}$ & $\begin{array}{c}\text { Line Flux } \\
\left(\text { ergs } \mathrm{cm}^{-2} \mathrm{~s}^{-1}\right)\end{array}$ & $\begin{array}{c}\text { Continuum } \\
\left(\operatorname{ergs~} \mathrm{cm}^{-2} \mathrm{~s}^{-1} \AA^{-1}\right)\end{array}$ \\
\hline & & \multicolumn{3}{|c|}{05 September 2010} & \multicolumn{3}{|c|}{25 November 2010} \\
\hline O I & 8446 & +1.0 & $3.19(-14)$ & $3.19(-14)$ & $\ldots$ & $\ldots$ & $\ldots$ \\
\hline Fe II & 8448 & $\ldots$ & $\ldots$ & $\ldots$ & -1.2 & $7.24(-16)$ & $6.03(-16)$ \\
\hline $\mathrm{H} \mathrm{I}(\mathrm{Pa} 17)$ & 8467 & -1.1 & $3.56(-14)$ & $3.24(-14)$ & -1.8 & $1.11(-15)$ & $6.14(-16)$ \\
\hline Ca II & 8498 & -15.4 & $5.08(-13)$ & $3.30(-14)$ & -15.6 & $9.83(-15)$ & $6.30(-16)$ \\
\hline Fe I & 8514 & -2.1 & $6.98(-14)$ & $3.32(-14)$ & -2.9 & $1.85(-15)$ & $6.38(-16)$ \\
\hline $\mathrm{Ca}$ II & 8543 & -13.6 & $4.57(-13)$ & $3.36(-14)$ & -12.6 & $8.20(-15)$ & $6.51(-16)$ \\
\hline Fe I & 8611 & -0.8 & $2.73(-14)$ & $3.41(-14)$ & -4.3 & $2.91(-15)$ & $6.76(-16)$ \\
\hline $\mathrm{Ca} \mathrm{II} / \mathrm{Fe} \mathrm{I}^{\mathrm{c}}$ & 8662 & -10.5 & $3.58(-13)$ & $3.41(-14)$ & -9.6 & $6.59(-15)$ & $6.87(-16)$ \\
\hline Fe I & 8674 & -1.2 & $4.08(-14)$ & $3.40(-14)$ & -1.4 & $9.63(-16)$ & $6.88(-16)$ \\
\hline Fe I & 8689 & -2.1 & $7.13(-14)$ & $3.39(-14)$ & -2.8 & $1.93(-15)$ & $6.89(-16)$ \\
\hline Mg I & 8807 & -3.6 & $1.18(-13)$ & $3.27(-14)$ & -3.6 & $2.42(-15)$ & $6.73(-16)$ \\
\hline Fe I & 8824 & -2.1 & $6.82(-14)$ & $3.25(-14)$ & -3.4 & $2.28(-15)$ & $6.72(-16)$ \\
\hline Fe I & 8838 & -1.1 & $3.55(-14)$ & $3.23(-14)$ & -1.2 & $8.05(-16)$ & $6.71(-16)$ \\
\hline
\end{tabular}

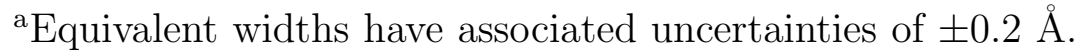

${ }^{\mathrm{b}}$ Number in parentheses is exponent e.g. $1.53(-14)$ means $1.53 \times 10^{-14}$.

${ }^{\mathrm{c}}$ Indicates blended line.

d... means line not present. 
${ }^{\mathrm{e}}$ Question mark indicates uncertain identification with strongest/most likely line listed.

${ }^{\mathrm{f}}$ Unresolved triplet. 
Table 4. NIR Spectral Features with $\mathrm{EW} \geq 1 \AA$ on One of the Two Observation Dates

\begin{tabular}{|c|c|c|c|c|c|c|c|}
\hline Line & $\begin{array}{c}\lambda \\
(\AA)\end{array}$ & $\begin{array}{c}\mathrm{EW}^{\mathrm{a}} \\
(\AA)\end{array}$ & $\begin{array}{c}\text { Line Flux } \\
\left(\text { ergs } \mathrm{cm}^{-2} \mathrm{~s}^{-1}\right) \\
02 \text { Octob }\end{array}$ & $\begin{array}{l}\quad \text { Continuum } \\
\left(\operatorname{ergs~} \mathrm{cm}^{-2} \mathrm{~s}^{-1} \AA^{-1}\right) \\
\text { r } 2010\end{array}$ & $\begin{array}{c}\mathrm{EW}^{\mathrm{a}} \\
(\AA)\end{array}$ & $\begin{array}{c}\text { Line Flux } \\
\left(\text { ergs } \mathrm{cm}^{-2} \mathrm{~s}^{-1}\right) \\
26 \text { Novem }\end{array}$ & $\begin{array}{l}\quad \text { Continuum } \\
\left(\operatorname{ergs~} \mathrm{cm}^{-2} \mathrm{~s}^{-1} \AA^{-1}\right) \\
\text { er } 2010\end{array}$ \\
\hline Fe I & 1.1599 & -2.0 & $1.6(-14)^{b}$ & $8.0(-15)$ & $n a^{c}$ & na & na \\
\hline Fe I & 1.1613 & -3.2 & $2.5(-14)$ & $8.1(-15)$ & na & na & na \\
\hline Fe I & 1.1644 & -2.4 & $2.0(-14)$ & $8.1(-15)$ & na & na & na \\
\hline K I & 1.1695 & -2.7 & $2.1(-14)$ & $8.0(-15)$ & na & na & na \\
\hline C I?? & 1.1758 & -1.1 & $9.3(-15)$ & $8.1(-15)$ & na & na & na \\
\hline Fe I & 1.1788 & -2.3 & $1.9(-14)$ & $8.3(-15)$ & na & na & na \\
\hline Mg I & 1.1832 & -3.3 & $2.7(-14)$ & $8.3(-15)$ & na & na & na \\
\hline Fe I & 1.1843 & -1.2 & $1.0(-14)$ & $8.2(-15)$ & na & na & na \\
\hline Fe I & 1.1887 & -5.5 & $4.6(-14)$ & $8.4(-15)$ & na & na & na \\
\hline$? ?$ & 1.1953 & -1.5 & $1.2(-14)$ & $8.3(-15)$ & na & na & na \\
\hline $\mathrm{Fe} I$ & 1.1976 & -4.3 & $3.7(-14)$ & $8.5(-15)$ & na & na & na \\
\hline Fe I & 1.1987 & -1.7 & $1.5(-14)$ & $8.6(-15)$ & na & na & na \\
\hline Si I?? & 1.1995 & -1.2 & $1.0(-14)$ & $8.6(-15)$ & na & na & na \\
\hline Fe I & 1.2035 & -2.4 & $2.0(-14)$ & $8.5(-15)$ & na & na & na \\
\hline $\mathrm{Fe} I$ & 1.2086 & -1.3 & $1.1(-14)$ & $8.3(-15)$ & na & na & na \\
\hline Si I?? & 1.2106 & -1.6 & $1.3(-14)$ & $8.3(-15)$ & na & na & na \\
\hline Fe I & 1.2273 & -1.6 & $1.4(-15)$ & $8.5(-15)$ & na & na & na \\
\hline $\mathrm{H} \mathrm{I} \mathrm{Pa} \beta$ & 1.2822 & -11.8 & $1.1(-13)$ & $9.2(-15)$ & na & na & na \\
\hline Fe I & 1.2902 & -1.0 & $9.1(-15)$ & $9.1(-15)$ & na & na & na \\
\hline Fe I & 1.3136 & -1.7 & $1.6(-14)$ & $9.4(-15)$ & na & na & na \\
\hline Si I?? & 1.3154 & -1.5 & $1.4(-14)$ & $9.5(-15)$ & na & na & na \\
\hline
\end{tabular}


Table 4-Continued

\begin{tabular}{|c|c|c|c|c|c|c|c|}
\hline Line & $\begin{array}{c}\lambda \\
(\AA)\end{array}$ & $\begin{array}{l}\mathrm{EW}^{\mathrm{a}} \\
(\AA)\end{array}$ & $\begin{array}{c}\text { Line Flux } \\
\left(\text { ergs } \mathrm{cm}^{-2} \mathrm{~s}^{-1}\right) \\
02 \text { Octol }\end{array}$ & $\begin{array}{l}\quad \text { Continuum } \\
\left(\operatorname{ergs~} \mathrm{cm}^{-2} \mathrm{~s}^{-1} \AA^{-1}\right) \\
2010\end{array}$ & $\begin{array}{l}\mathrm{EW}^{\mathrm{a}} \\
(\AA)\end{array}$ & $\begin{array}{c}\text { Line Flux } \\
\left(\operatorname{ergs~} \mathrm{cm}^{-2} \mathrm{~s}^{-1}\right) \\
26 \text { Novem }\end{array}$ & $\begin{array}{l}\quad \text { Continuum } \\
\left(\operatorname{ergs~cm}{ }^{-2} \mathrm{~s}^{-1} \AA^{-1}\right) \\
\text { er } 2010\end{array}$ \\
\hline Fe I & 1.3292 & -1.2 & $1.2(-14)$ & $9.7(-15)$ & na & na & na \\
\hline Fe I & 1.4885 & -2.4 & $3.3(-14)$ & $1.4(-14)$ & -2.1 & $4.7(-15)$ & $2.2(-15)$ \\
\hline Fe I & 1.5031 & -4.3 & $6.0(-14)$ & $1.4(-14)$ & -3.8 & $8.6(-15)$ & $2.3(-15)$ \\
\hline Mg I & 1.5046 & -3.3 & $4.5(-14)$ & $1.4(-14)$ & -3.2 & $7.2(-15)$ & $2.3(-15)$ \\
\hline $\mathrm{Fe} I$ & 1.5055 & -2.6 & $3.5(-14)$ & $1.4(-14)$ & -2.7 & $6.2(-15)$ & $2.3(-15)$ \\
\hline $\mathrm{Fe} I$ & 1.5300 & -1.0 & $1.4(-14)$ & $1.4(-14)$ & $\ldots{ }^{\mathrm{d}}$ & $\ldots$ & $\ldots$ \\
\hline H I Br 15 & 1.5560 & -1.1 & $1.6(-14)$ & $1.4(-14)$ & -1.2 & $3.0(-15)$ & $2.5(-15)$ \\
\hline Fe I & 1.5753 & -1.2 & $1.7(-14)$ & $1.5(-14)$ & -1.3 & $3.4(-15)$ & $2.6(-15)$ \\
\hline $\mathrm{Fe} I$ & 1.5771 & -2.1 & $3.1(-14)$ & $1.5(-14)$ & -1.9 & $5.0(-15)$ & $2.7(-15)$ \\
\hline H I Br 14 & 1.5883 & -1.2 & $1.7(-14)$ & $1.5(-14)$ & $\ldots$ & $\ldots$ & $\ldots$ \\
\hline Si I & 1.5892 & -2.4 & $3.6(-14)$ & $1.5(-14)$ & -2.1 & $5.7(-15)$ & $2.7(-15)$ \\
\hline Fe I & 1.5963 & -1.1 & $1.6(-14)$ & $1.5(-14)$ & $\ldots$ & $\ldots$ & $\ldots$ \\
\hline H I Br 13 & 1.6111 & -1.1 & $1.7(-14)$ & $1.5(-14)$ & $\ldots$ & $\ldots$ & $\ldots$ \\
\hline H I Br 12 & 1.6409 & -1.7 & $2.6(-14)$ & $1.5(-14)$ & -1.7 & $5.1(-15)$ & $3.0(-15)$ \\
\hline [Fe II] & 1.6432 & $\ldots$ & $\ldots$ & $\ldots$ & -1.2 & $3.6(-15)$ & $3.0(-15)$ \\
\hline H I Br 11 & 1.6808 & -1.9 & $3.0(-14)$ & $1.6(-14)$ & -1.8 & $5.6(-15)$ & $3.1(-15)$ \\
\hline Mg I & 1.7112 & -2.0 & $3.3(-14)$ & $1.7(-14)$ & -1.6 & $5.5(-15)$ & $3.4(-15)$ \\
\hline H I Br 10 & 1.7366 & -2.4 & $4.2(-14)$ & $1.8(-14)$ & -2.4 & $8.8(-15)$ & $3.7(-15)$ \\
\hline Fe I & 1.7477 & -0.6 & $1.1(-14)$ & $1.8(-14)$ & -1.2 & $4.4(-15)$ & $3.8(-15)$ \\
\hline$[\mathrm{Fe} \mathrm{II}]$ & 2.0152 & +1.8 & $3.0(-14)$ & $1.7(-14)$ & $\ldots$ & $\ldots$ & $\ldots$ \\
\hline Fe I & 2.0336 & $\ldots$ & $\ldots$ & $\ldots$ & -1.1 & $5.4(-15)$ & $4.8(-15)$ \\
\hline
\end{tabular}


Table 4-Continued

\begin{tabular}{|c|c|c|c|c|c|c|c|}
\hline Line & $\begin{array}{c}\lambda \\
(\AA)\end{array}$ & $\begin{array}{l}\mathrm{EW}^{\mathrm{a}} \\
(\AA)\end{array}$ & $\begin{array}{c}\text { Line Flux } \\
\left(\operatorname{ergs} \mathrm{cm}^{-2} \mathrm{~s}^{-1}\right) \\
02 \text { Octol }\end{array}$ & $\begin{array}{l}\quad \text { Continuum } \\
\left(\operatorname{ergs~} \mathrm{cm}^{-2} \mathrm{~s}^{-1} \AA^{-1}\right) \\
2010\end{array}$ & $\begin{array}{l}\mathrm{EW}^{\mathrm{a}} \\
(\AA)\end{array}$ & $\begin{array}{c}\text { Line Flux } \\
\left(\operatorname{ergs} \mathrm{cm}^{-2} \mathrm{~s}^{-1}\right) \\
26 \text { Novem }\end{array}$ & $\begin{array}{l}\text { Continuum } \\
\left(\operatorname{ergs~} \mathrm{cm}^{-2} \mathrm{~s}^{-1} \AA^{-1}\right) \\
\text { er } 2010\end{array}$ \\
\hline $\mathrm{H}_{2} 1-0 \mathrm{~S}(1)$ & 2.1214 & -0.7 & $1.3(-14)$ & $1.7(-14)$ & -3.0 & $1.5(-14)$ & $5.2(-15)$ \\
\hline $\mathrm{H} \mathrm{I}(\mathrm{Br} \gamma)$ & 2.1658 & -5.1 & $9.0(-14)$ & $1.8(-14)$ & -4.2 & $2.3(-14)$ & $5.5(-15)$ \\
\hline $\mathrm{Na} \mathrm{I}$ & 2.2061 & -1.1 & $1.9(-14)$ & $1.8(-14)$ & -0.8 & $4.5(-15)$ & $5.8(-15)$ \\
\hline $\mathrm{NaI}$ & 2.2087 & -1.0 & $1.8(-14)$ & $1.8(-14)$ & -0.7 & $4.0(-15)$ & $5.8(-15)$ \\
\hline $\mathrm{H}_{2} 1-0 \mathrm{~S}(0)^{\mathrm{e}}$ & 2.2235 & $\ldots$ & $\ldots$ & $\ldots$ & -0.7 & $4.4(-15)$ & $5.9(-15)$ \\
\hline $\mathrm{H}_{2} 2-1 \mathrm{~S}(1)^{\mathrm{e}}$ & 2.2477 & $\ldots$ & $\ldots$ & $\ldots$ & -0.5 & $2.9(-15)$ & $6.1(-15)$ \\
\hline $\mathrm{CO} \mathrm{v}=2-0$ & $2.2931^{\mathrm{f}}$ & -15.1 & $2.9(-13)$ & $1.9(-14)$ & -10.7 & $6.9(-14)$ & $6.4(-15)$ \\
\hline
\end{tabular}

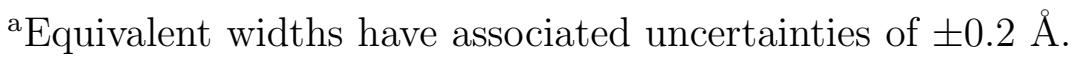

${ }^{\mathrm{b}}$ Number in parentheses is exponent e.g. $1.53(-14)$ means $1.53 \times 10^{-14}$.

${ }^{c}$ na means data not available.

d... means line not present.

eine included even though it has an $\mathrm{EW}<1 \AA$ since it is useful for comparative purposes.

${ }^{\mathrm{f}} \mathrm{EW}$ measured from $2.2930-2.3000 \mu \mathrm{m}$. 
Table 5. Pre-Outburst Photometry of V2492 Cyg

\begin{tabular}{|c|c|c|c|}
\hline $\begin{array}{l}\text { Wavelength } \\
\qquad(\mu \mathrm{m})\end{array}$ & $\begin{array}{l}\text { Origin } \\
\text { of Flux }\end{array}$ & $\begin{array}{l}\text { Epoch } \\
\text { of Flux }\end{array}$ & $\begin{array}{l}\text { Flux } \\
\text { (mJy) }\end{array}$ \\
\hline 1.25 & UKIDSS & 2006.7 & $0.03 \pm 0.003$ \\
\hline 1.65 & UKIDSS & 2006.7 & $0.25 \pm 0.025$ \\
\hline 2.20 & UKIDSS & 2006.7 & $3.8 \pm 0.38$ \\
\hline 3.6 & Spitzer/IRAC & 2006.6 & $74 \pm 7$ \\
\hline 4.5 & Spitzer/IRAC & 2006.6 & $158 \pm 16$ \\
\hline 5.6 & Spitzer/IRAC & 2006.6 & $653 \pm 65$ \\
\hline 8.0 & Spitzer/IRAC & 2006.6 & $1200 \pm 120$ \\
\hline 9.0 & $A K A R I / I R C$ & 2006.6 & $1960 \pm 200$ \\
\hline 18.0 & AKARI/IRC & 2006.6 & $3875 \pm 457$ \\
\hline 24.0 & Spitzer/MIPS & 2006.6 & $3402 \pm 340$ \\
\hline 70.0 & Spitzer/MIPS & 2006.6 & $5880 \pm 588$ \\
\hline 1100.0 & Bolocam GPS & 2009.4 & $148 \pm 74$ \\
\hline \multicolumn{4}{|c|}{ Additional Photometry } \\
\hline 8.28 & $M S X 6 C$ & 1996.6 & $1610 \pm 66$ \\
\hline 12.0 & $I R A S$ & 1983 & $3390 \pm 330$ \\
\hline 12.13 & $M S X 6 C$ & 1996.6 & $2490 \pm 137$ \\
\hline 14.65 & $M S X 6 C$ & 1996.6 & $3045 \pm 189$ \\
\hline 21.34 & $M S X 6 C$ & 1996.6 & $3140 \pm 201$ \\
\hline 25.0 & $I R A S$ & 1983 & $6590 \pm 660$ \\
\hline 60.0 & $\operatorname{IRAS}$ & 1983 & $27890 \pm 1000$ \\
\hline 100.0 & $\operatorname{IRAS}$ & 1983 & $57350 \pm 1000$ \\
\hline
\end{tabular}


Table 6. SED Fitting Best-Fit Parameters

\begin{tabular}{|c|c|c|c|c|c|}
\hline $\begin{array}{c}\text { Parameter } \\
\text { Name }\end{array}$ & Units & $\begin{array}{l}\text { Best-Fit }^{\mathrm{a}} \\
\text { Value }\end{array}$ & $\begin{array}{c}\text { Best-Fit }^{\mathrm{b}} \\
\text { Value }\end{array}$ & $\begin{array}{l}\text { Best-Fit }^{\mathrm{c}} \\
\text { Value }\end{array}$ & $\begin{array}{l}\text { Best-Fit }^{\mathrm{d}} \\
\text { Value }\end{array}$ \\
\hline Model \# & & 3009214 & 3019063 & 3015948 & Top 20 \\
\hline$\chi^{2}$ & & 274 & 602 & 1519 & $795 \pm 14$ \\
\hline Mass & $\left(\mathrm{M}_{\odot}\right)$ & 3.5 & 0.7 & 1.9 & $0.52 \pm 0.43$ \\
\hline Temperature & $(\mathrm{K})$ & 13000 & 3800 & 4400 & $3580 \pm 85$ \\
\hline Age & (yrs) & $4 \times 10^{6}$ & $1.6 \times 10^{3}$ & $1.5 \times 10^{5}$ & $2.1 \pm 0.2 \times 10^{3}$ \\
\hline $\mathrm{R}_{*}{ }^{\mathrm{e}}$ & $\left(\mathrm{R}_{\odot}\right)$ & 2 & 9 & 10 & $6.9 \pm 0.3$ \\
\hline $\mathrm{L}_{t o t}{ }^{\mathrm{f}}$ & $\left(\mathrm{L}_{\odot}\right)$ & 126 & 14 & 30 & $17 \pm 2$ \\
\hline Distance & (pc) & 500 & 500 & 500 & $521 \pm 8$ \\
\hline Inclination $^{\mathrm{g}}$ & (degs) & 31.8 & 31.8 & 31.8 & $50 \pm 3$ \\
\hline$\dot{\mathrm{M}}_{e n v} \mathrm{~h}$ & $\left(\mathrm{M}_{\odot} \mathrm{yr}^{-1}\right)$ & 0 & $4 \times 10^{-6}$ & $3 \times 10^{-5}$ & $3.6 \pm 0.3 \times 10^{-6}$ \\
\hline$\dot{\mathrm{M}}_{d i s k}^{\mathrm{i}}$ & $\left(\mathrm{M}_{\odot} \mathrm{yr}^{-1}\right)$ & $8 \times 10^{-8}$ & $9 \times 10^{-8}$ & $3 \times 10^{-9}$ & $5.2 \pm 1.1 \times 10^{-6}$ \\
\hline $\mathrm{A}_{V}(\mathrm{int})^{\mathrm{j}}$ & (mags) & 35 & 12 & 25 & $7 \pm 0.5$ \\
\hline $\mathrm{A}_{V}(\mathrm{cir})^{\mathrm{k}}$ & (mags) & 0 & 57 & 26 & $96 \pm 8$ \\
\hline $\mathrm{M}_{e n v} 1$ & $\left(\mathrm{M}_{\odot}\right)$ & $8 \times 10^{-9}$ & $1 \times 10^{-2}$ & $4 \times 10^{-1}$ & $1.8 \pm 0.2 \times 10^{-2}$ \\
\hline $\mathrm{M}_{\text {disk }}{ }^{\mathrm{m}}$ & $\left(\mathrm{M}_{\odot}\right)$ & $6 \times 10^{-3}$ & $1 \times 10^{-2}$ & $8 \times 10^{-4}$ & $8.4 \pm 1.3 \times 10^{-3}$ \\
\hline
\end{tabular}

${ }^{a}$ Unconstrained best-fit values.

${ }^{\mathrm{b}}$ Best-fit values constraining $\mathrm{M}_{*} \leq 2 \mathrm{M}_{\odot}$.

${ }^{\text {c} B e s t-f i t ~ v a l u e s ~ c o n s t r a i n i n g ~} \mathrm{M}_{*} \leq 2 \mathrm{M}_{\odot}$ and age between $10^{5}$ and $10^{7}$ yrs.

${ }^{\mathrm{d}}$ Values obtained from averaging the top 20 best-fit models with $\mathrm{A}_{V}$ (interstellar) $<10$ mags.

${ }^{e}$ Radius of star.

${ }^{\mathrm{f}}$ Total luminosity $=$ star+accretion luminosities.

${ }^{g}$ Required disk inclination with respect to the line-of-sight.

${ }^{\mathrm{h}}$ Mass accretion rate from envelope to disk. 
${ }^{i}$ Mass accretion rate from disk to stellar surface.

${ }^{j}$ Visual extinction not associated with circumstellar disk and envelope.

${ }^{\mathrm{k}}$ Visual extinction from circumstellar disk and envelope.

${ }^{l}$ Envelope mass.

${ }^{m}$ Disk mass. 
Table 7. Accretion Luminosity/Rates from Br $\gamma$ Emission Line Fluxes

\begin{tabular}{lccccc}
\hline \hline UT Date & $\begin{array}{c}\text { Flux }(\text { observed })^{\mathrm{a}} \\
\left(\mathrm{ergs} \mathrm{cm}{ }^{-2} \mathrm{~s}^{-1}\right)\end{array}$ & $\begin{array}{c}\mathrm{A}_{V}^{\mathrm{b}} \\
(\mathrm{mags})\end{array}$ & $\begin{array}{c}\text { Flux }(\text { dereddened })^{\mathrm{c}} \\
\left(\mathrm{ergs} \mathrm{cm}^{-2} \mathrm{~s}^{-1}\right)\end{array}$ & $\begin{array}{c}\mathrm{L}_{a c c}{ }^{\mathrm{d}} \\
\left(\mathrm{L}_{\odot}\right)\end{array}$ & $\begin{array}{c}\dot{\mathrm{M}}_{a c c} \mathrm{e} \\
\left(\mathrm{M}_{\odot} \mathrm{yr}^{-1}\right)\end{array}$ \\
\hline 2010 July 14 & $1.4(-14)^{\mathrm{g}}$ & 12 & $4.8(-14)$ & 2.1 & $6.4(-7)$ \\
2010 July 18 & $2.9(-14)$ & 12 & $1.0(-13)$ & 5.2 & $1.6(-6)$ \\
2010 October 2 & $9.0(-14)$ & 2 & $1.1(-13)$ & 5.9 & $1.8(-6)$ \\
2010 November 26 & $2.3(-14)$ & 7 & $4.7(-14)$ & 2.0 & $6.3(-7)$ \\
\hline
\end{tabular}

${ }^{\mathrm{a} O b s e r v e d} \mathrm{Br} \gamma$ emission line flux.

${ }^{\mathrm{b}}$ Visual extinction from JHK c-c diagram.

${ }^{\mathrm{c}}$ Dereddened $\operatorname{Br} \gamma$ emission line flux.

${ }^{\mathrm{d}}$ Accretion luminosity.

${ }^{\mathrm{e}}$ Accretion rate.

${ }^{\mathrm{f}}$ From Covey et al. (2011).

${ }^{\mathrm{g}}$ Number in parentheses is exponent e.g. $1.4(-14)$ means $1.4 \times 10^{-14}$. 
Table 8. Mass-Loss Rates from [O I] Emission Line Fluxes

\begin{tabular}{lccccc}
\hline \hline UT Date & $\begin{array}{c}\text { Flux } \\
\left(\text { ergs cm }^{-2} \mathrm{~s}^{-1}\right)\end{array}$ & $\begin{array}{c}\mathrm{A}_{V}^{\mathrm{a}} \\
(\mathrm{mags})\end{array}$ & $\begin{array}{c}\mathrm{L}_{6300}(\text { observed })^{\mathrm{b}} \\
\left(\mathrm{L}_{\odot}\right)\end{array}$ & $\begin{array}{c}\mathrm{L}_{6300}(\text { dereddened })^{\mathrm{c}} \\
\left(\mathrm{L}_{\odot}\right)\end{array}$ & $\begin{array}{c}\dot{\mathrm{M}}_{\text {wind }} \\
\left(\mathrm{M}_{\odot} \mathrm{yr}^{-1}\right)\end{array}$ \\
\hline 2010 July 8 & $5.5(-15)^{\mathrm{e}}$ & 12 & $6.2(-5)$ & $4.4(-1)$ & $1.6(-6)$ \\
2010 July 19 & $4.0(-15)$ & 12 & $4.5(-5)$ & $3.3(-1)$ & $1.1(-6)$ \\
2010 September 5 & $1.4(-14)$ & 2 & $1.6(-4)$ & $7.1(-4)$ & $2.5(-9)$ \\
2010 November 25 & $4.9(-15)$ & 7 & $5.5(-5)$ & $1.0(-2)$ & $3.4(-8)$ \\
\hline
\end{tabular}

${ }^{a}$ Visual extinction from JHK c-c diagram.

${ }^{\mathrm{b}}$ Observed emission line luminosity.

${ }^{\mathrm{c}}$ Dereddened emission line luminosity.

${ }^{\mathrm{d}}$ From Covey et al. (2011).

e Number in parentheses is exponent e.g. 5.5(-15) means $5.5 \times 10^{-15}$. 


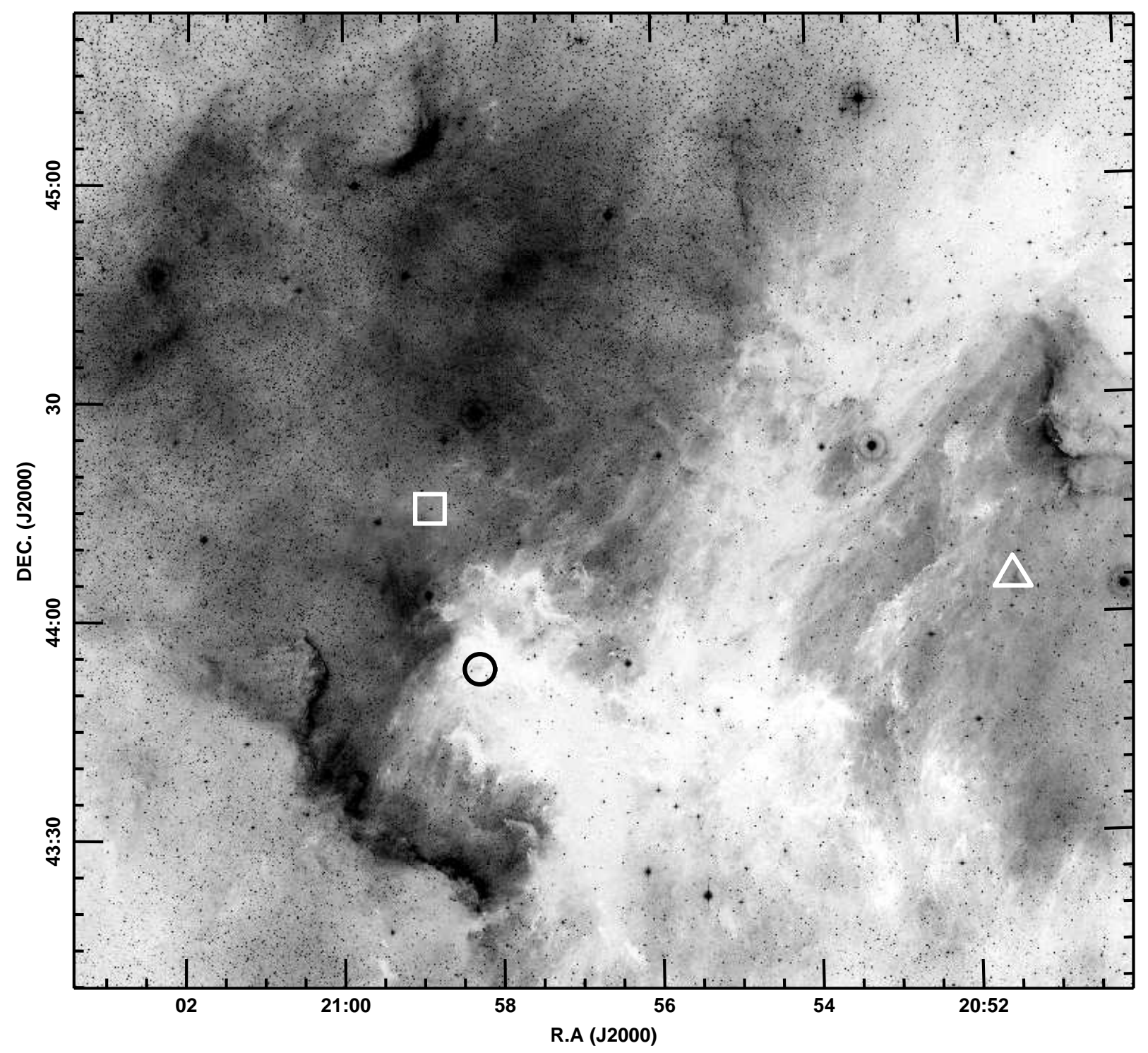

Fig. 1. - The DSS-2 red plate image of the region surrounding V2492 Cyg. The image is approximately 2 degrees square. The color table of the image has been reversed so that bright objects are black and dark object white and hence the dark clouds associated with this region are white. The location of V2492 Cyg is indicated by the white triangle. The white square indicates the classical FU Orionis eruptive variable V1057 Cyg while the black circle shows the second young star in the region that recently erupted, HBC 722. The bright complex (black) to the north is the North America nebula (NGC 7000) while the one to the west is the Pelican Nebula (IC 5070/5067). 

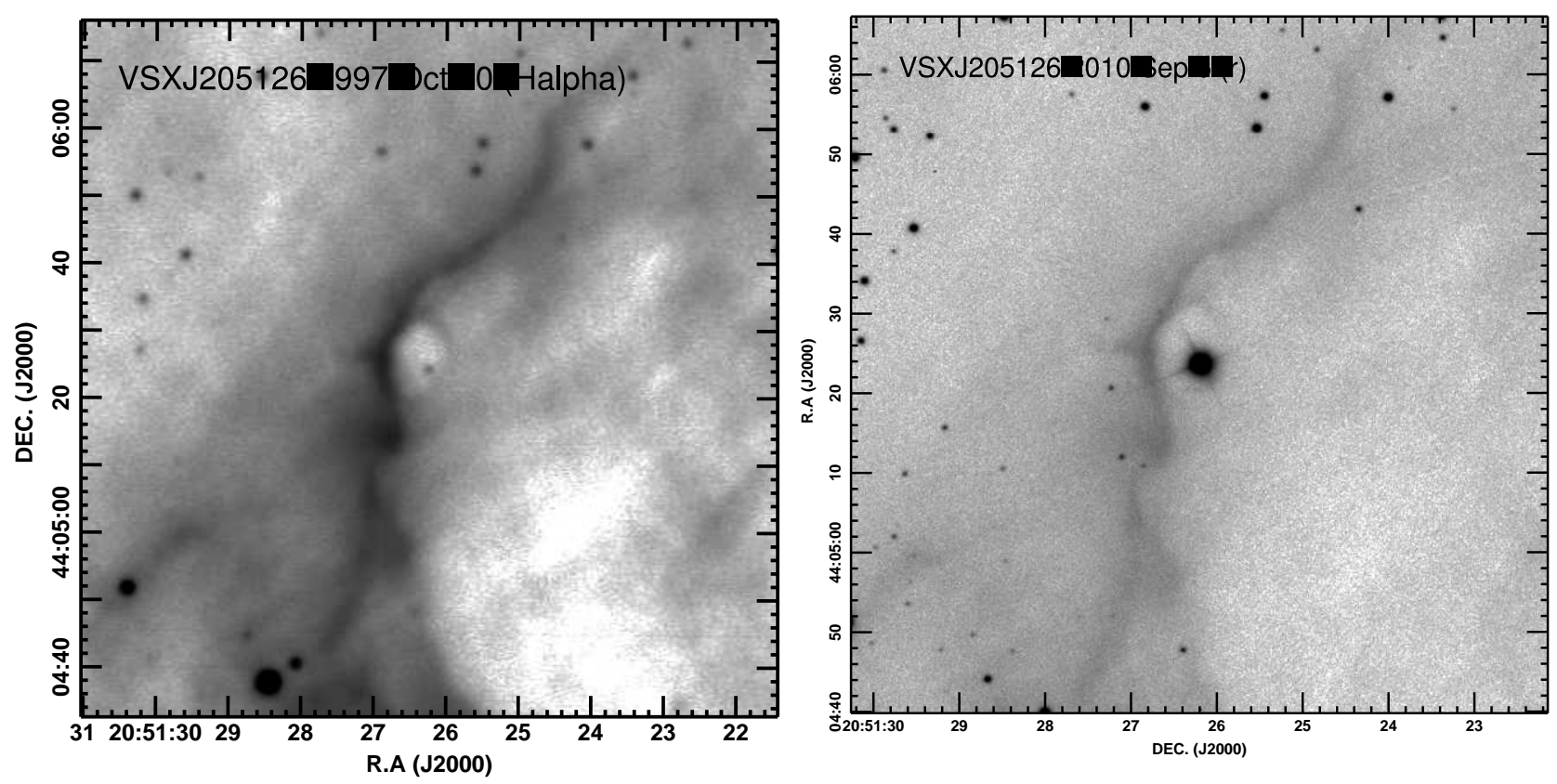

Fig. 2.- Optical images of V2492 Cyg. The left and right images are of V2492 Cyg (in the4 center of the image) from UT 1997 Oct 30 and UT 2010 Sep 5, respectively. The 1997 image is from Bally \& Reipurth (2003) and is an $\mathrm{H} \alpha$ image. The 2010 image is in r' and from Gemini-North. Note the significant brightness difference of V2492 Cyg. 

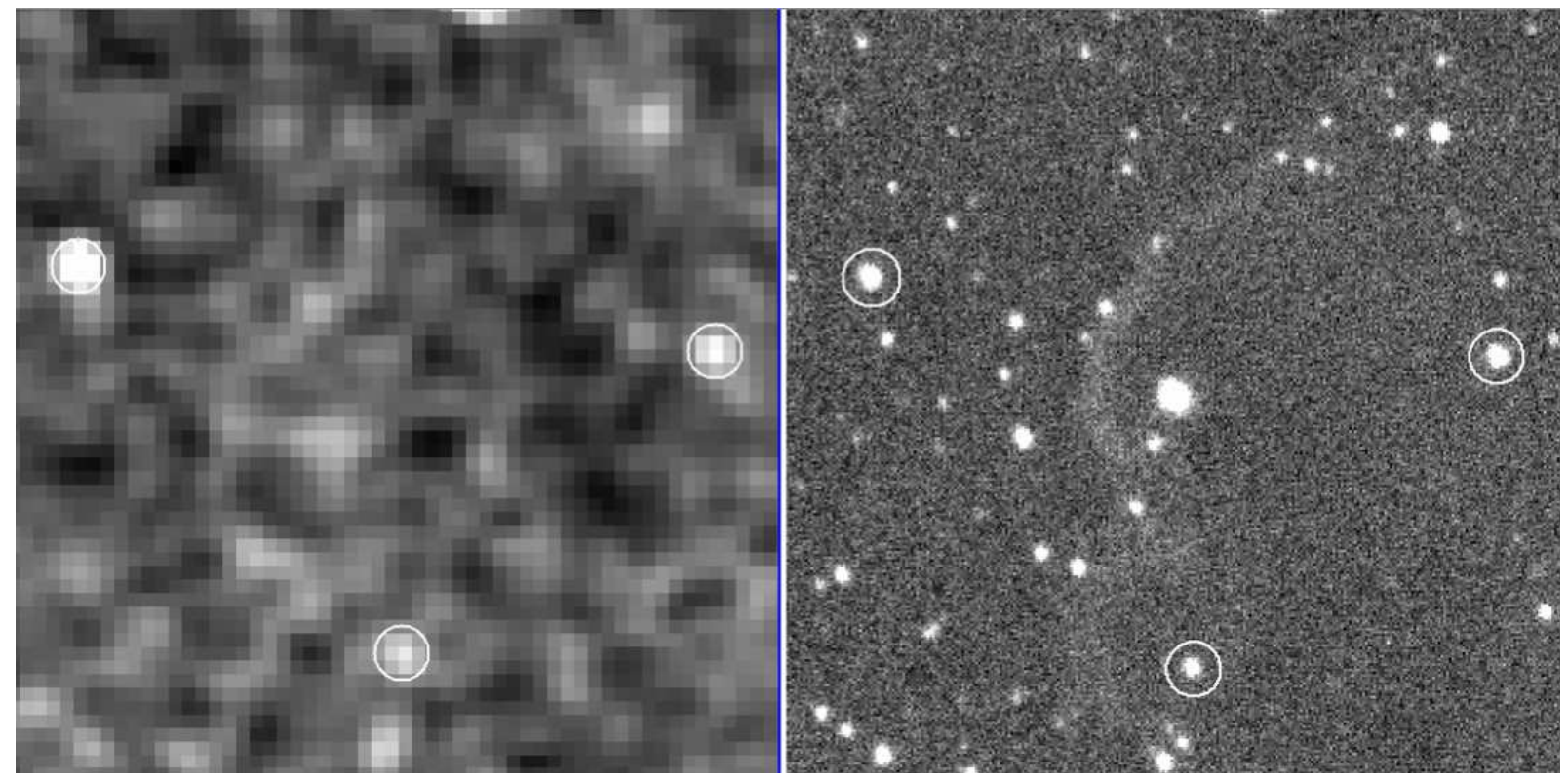

Fig. 3.- Comparison of the 2MASS K' image (left, taken in 2000) of the region containing V2492 Cyg (left) and the UKIRT UKIDSS K' image (right, taken in 2006). North is at the top, east to the left and each image is approximately $60^{\prime \prime}$ square. Common stars are indicated by white circles. Note that there is no sign of V2492 Cyg in the 2MASS images indicating that it is fainter than the $10 \sigma \mathrm{K}^{\prime}$ detection limit of 14.3 mags. For reference, the western of the circled stars has a K' magnitude of 14.7 mags. In the UKIDSS Galactic Plane Survey, V2492 Cyg has a K' magnitude of 13.15. 

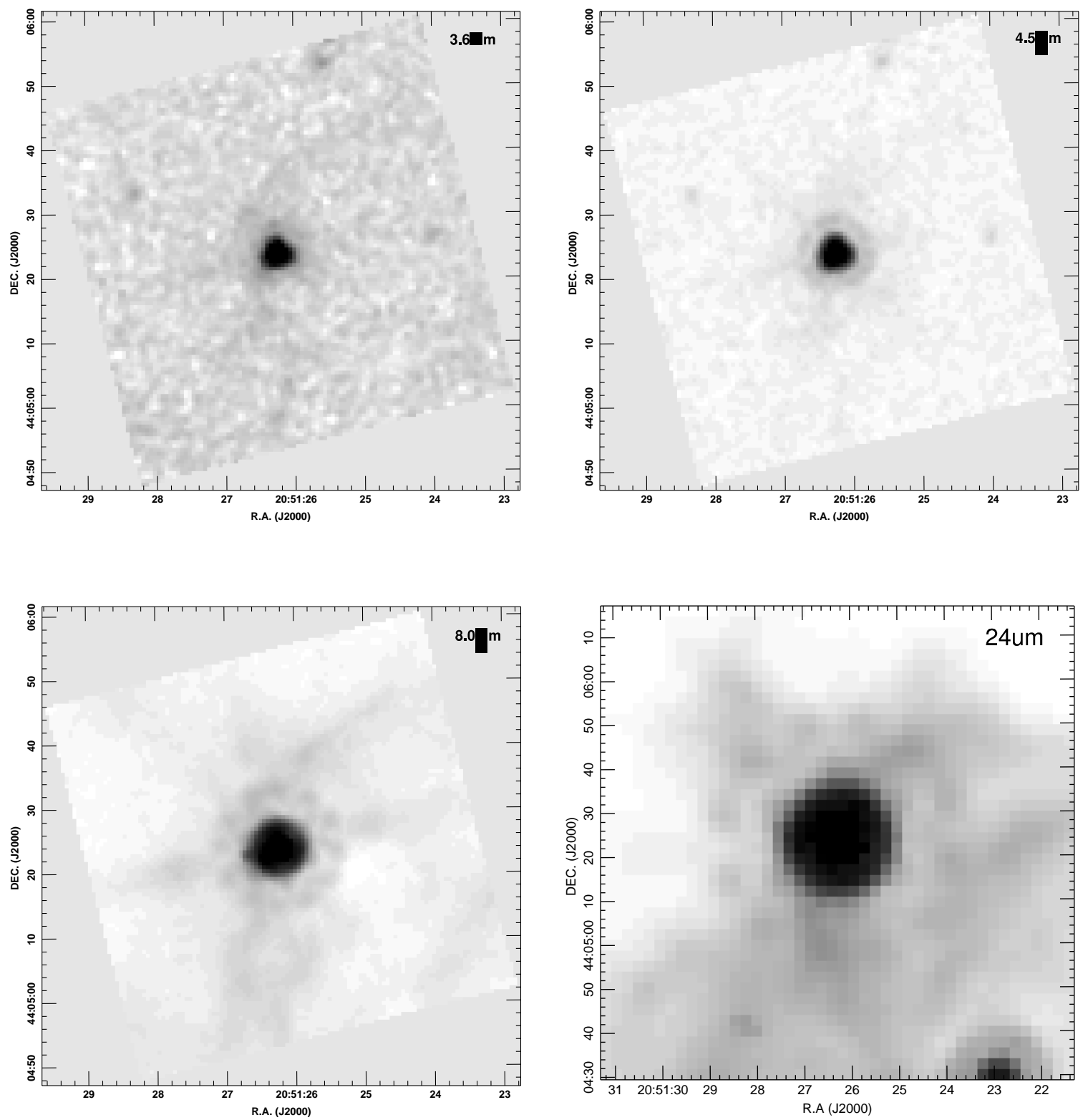

Fig. 4. - Spitzer IRAC and MIPS images of V2492 Cyg. These images are $72^{\prime \prime}$ square and are from the IRAC band $1[3.6 \mu \mathrm{m}]$ (top-left), the IRAC band $2[4.5 \mu \mathrm{m}]$ (top-right), the IRAC band $4[8.0 \mu \mathrm{m}]$ (bottom-left), the MIPS band 1 [24 $\mu \mathrm{m}]$ (bottom-right) images. V2492 Cyg is the bright point-source at the center of each image. The IRAC images were acquired on UT 2006 August 9 while the MIPS image was taken on UT 2006 June 10. 


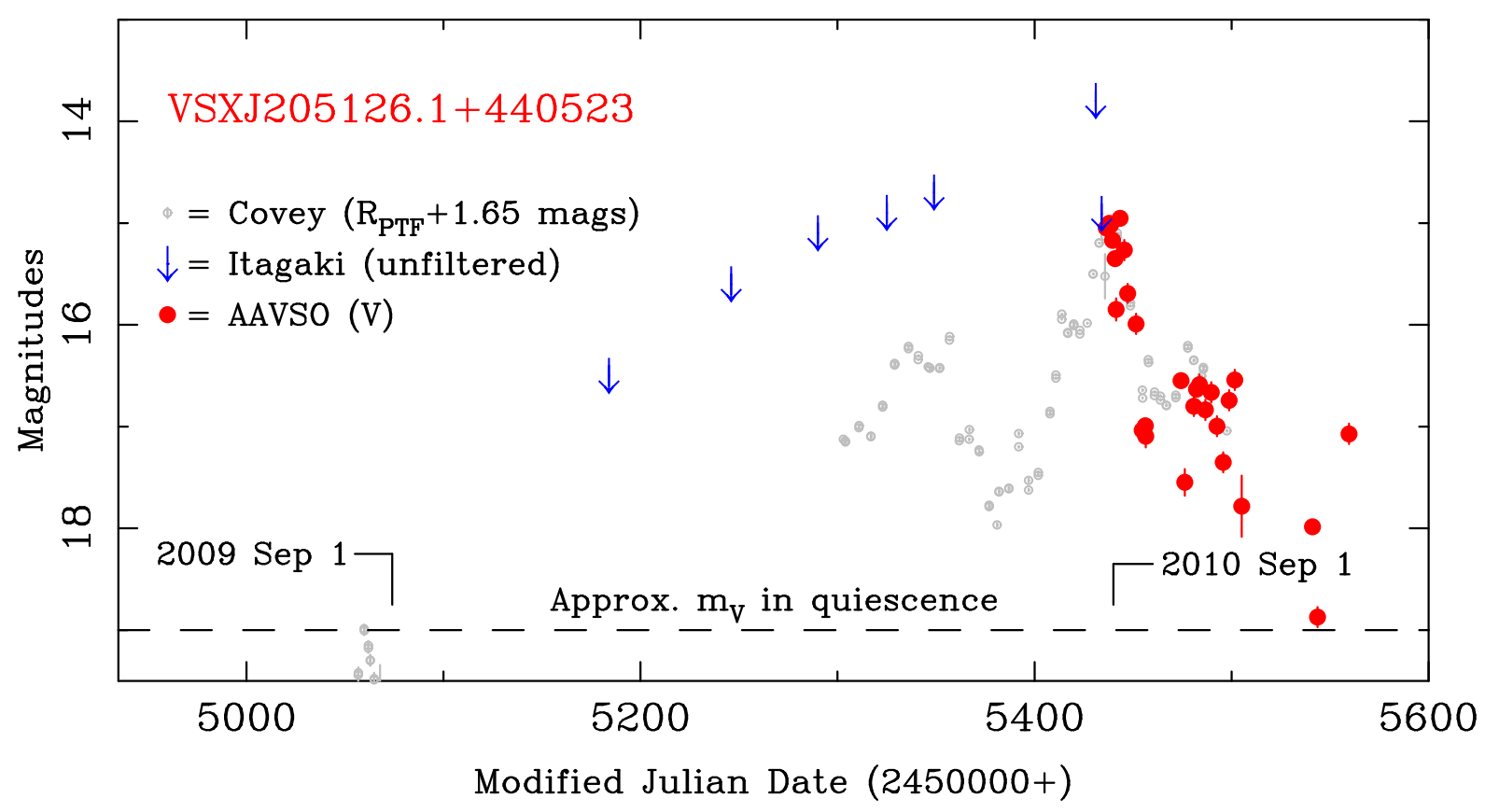

Fig. 5.- The optical V-band light-curve of V2492 Cyg using data from the AAVSO database. The plot is up to date to the time of paper submission. The quiescent V-band magnitude is shown as a horizontal dashed line. The AAVSO observations (red filled circles) have been averaged to produce one composite measurement per night. Error bars are shown on each point and are either calculated from the spread of values throughout each specific night or, in the case of only one observation per night, the quoted observational uncertainty in the AAVSO database. The downward facing blue arrows indicate points from Itagaki (2010) in unfiltered light and hence are upper-limits. The small open grey circles are the R-band data from Covey et al. (2011) shifted by +1.65 mags (to approximately the same level as the V-band measurements) and are shown to indicate the level of variability observed. 

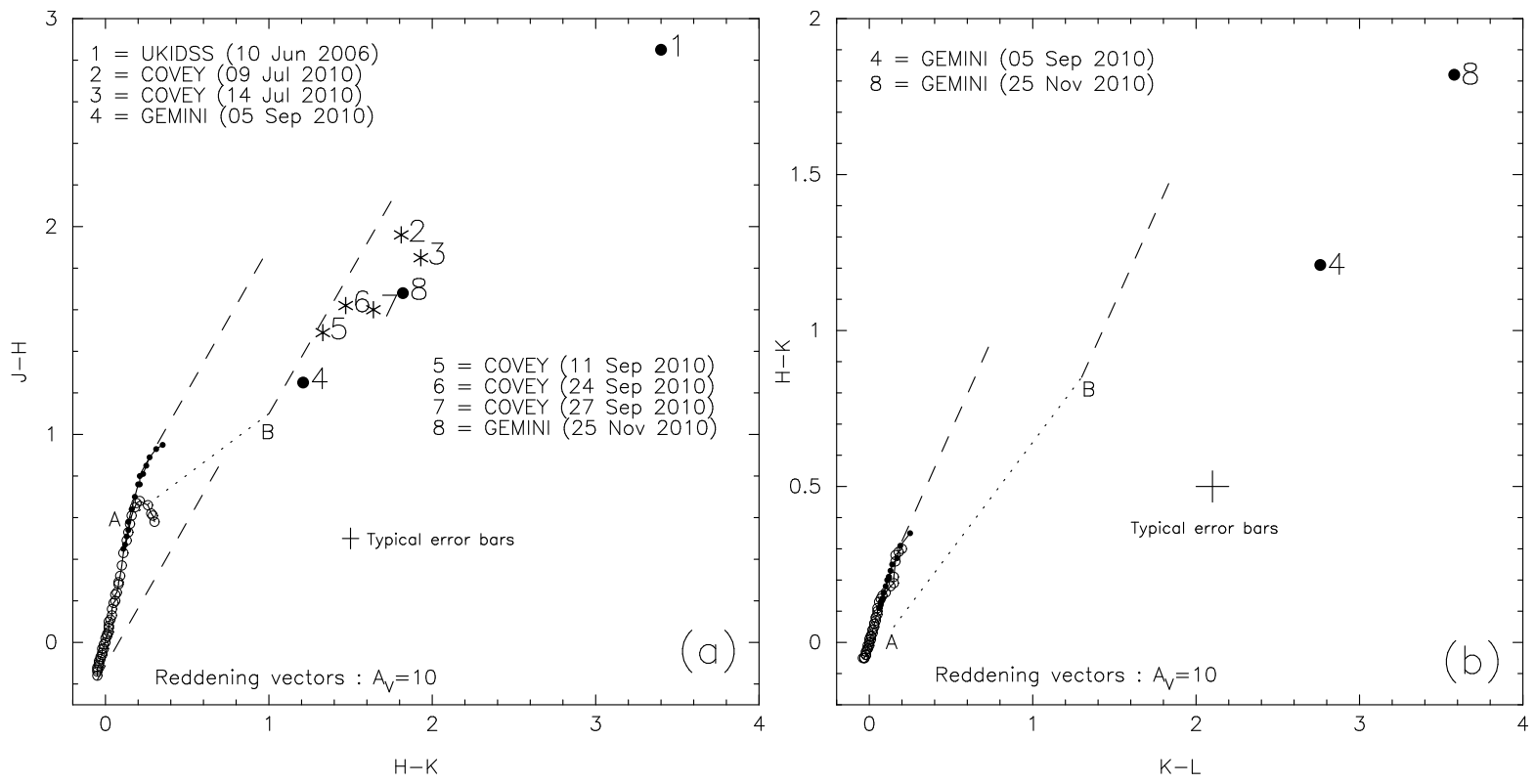

Fig. 6.- NIR color-color diagrams showing the location of V2492 Cyg. Left (a) shows the J-H vs. H-K' diagram and points \#1 and \#1 are V2492 Cyg from two epochs. On the right (b) is a H-K' vs. K'-L' plot showing V2492 Cyg from one epoch (\#2). Typical photometric uncertainties are shown and are of the order \pm 0.1 mags. The location of zero-age main sequence dwarfs and giants are shown in both plots. Reddening vectors extend from the extremes of the dwarf and giant loci with a length $A_{V}=10$ mags. The extinction law is from the Robitaille et al. (2007) SED models. The dashed lines show the Greene \& Meyer (1995) loci for classical and weak-line T Tauri stars. Extending from the end of these loci is an additional reddening vector (dot-dashed line). 


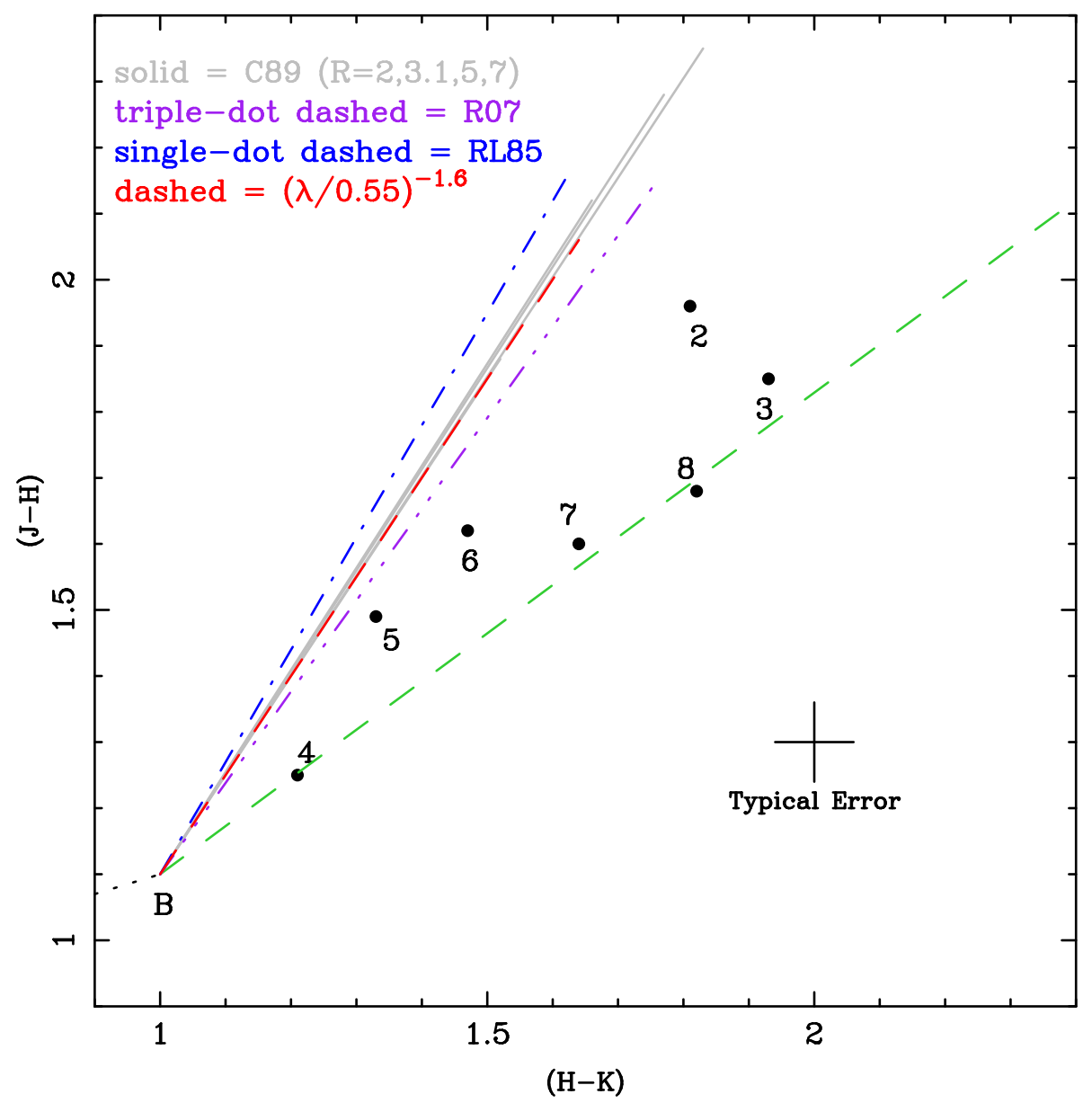

Fig. 7.- NIR JHK color-color diagram showing the progression of $\mathrm{J}-\mathrm{H}$ and $\mathrm{H}-\mathrm{K}$ color changes from point $\# 1$ to $\# 8$. Point \#1 is outside the plotted area and along the dashed (green) line. Also shown are a selection of reddening vectors representing different reddening laws. The grey lines show the Cardelli, Clayton, \& Mathis (1989) reddening law for four different values of $\mathrm{R}$, the ratio of total to selective extinction. The dashed (red) line shows a generic reddening law with an exponent of -1.6. The single-dot dashed (blue) line is the Rieke \& Lebofsky (1985) reddening law. Finally, the triple-dot dashed (purple) line shows the reddening law adopted by Robitaille et al. (2007) in their SED models. Although there is significant divergence of these reddening vectors at large $\mathrm{A}_{V}$, none alone can explain the changes observed in the V2492 Cyg observations. 

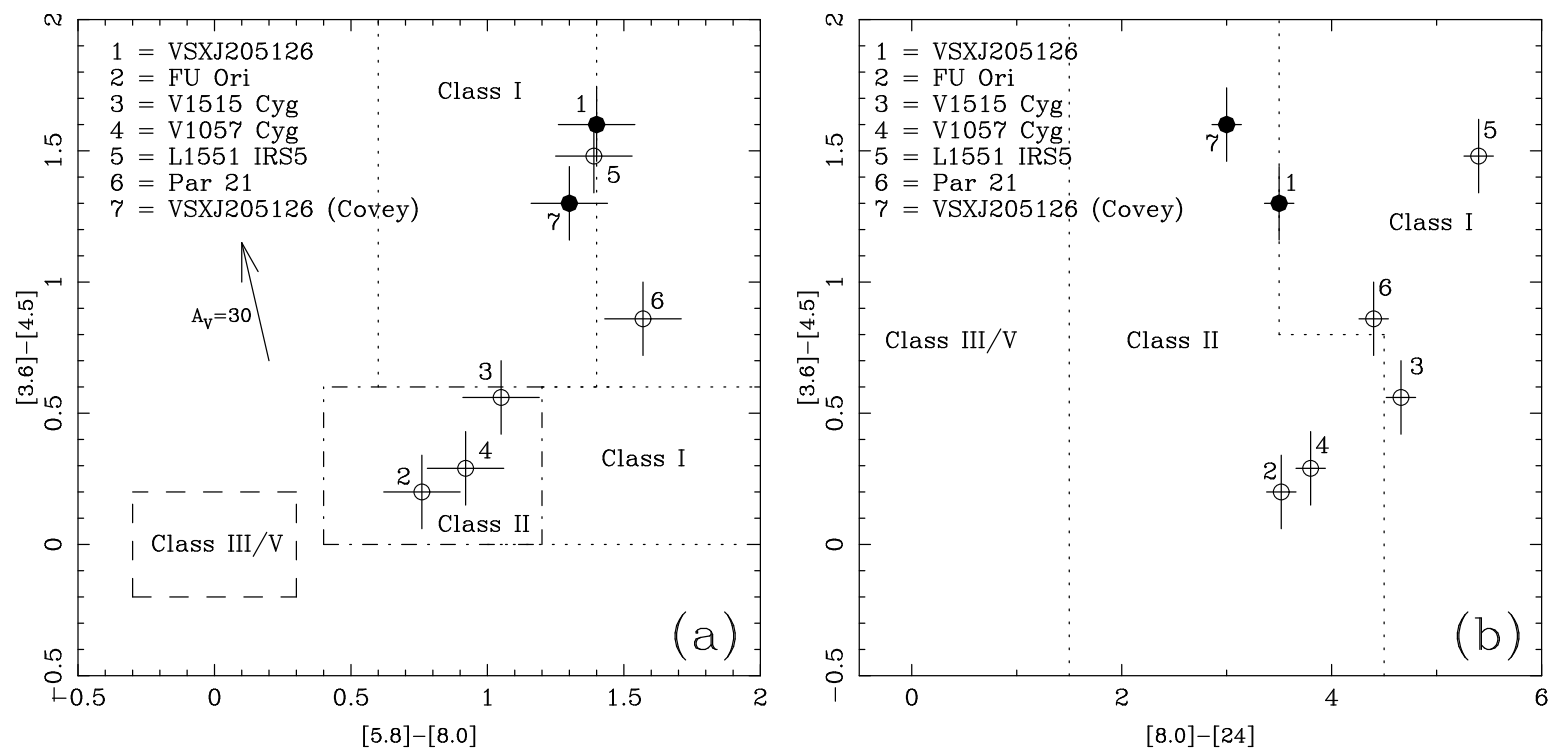

Fig. 8. - Spitzer IRAC and IRAC/MIPS color-color (c-c) diagrams showing the location of V2492 Cyg. Left (a) shows the [3.6]-[4.5] diagram and point \#2 (filled circles) is V2492 Cyg. Points \#2-6 (open circles) are the FUors/FUor-like objects FU Ori, V1515 Cyg, V1057 Cyg, L1551 IRS5, and Par 21, respectively. On the right (b) is a [3.6]-[4.5] vs. [8.0]-[24] plot with the same source identification numbers. Typical photometric uncertainties are shown and are of the order \pm 0.1 mags. The evolutionary class boundaries from Allen et al. (2002) are shown as are reddening vectors for $\mathrm{A}_{V}=30$ mags. V2492 Cyg is located well inside the Class I regime in the IRAC c-c diagram. In the IRAC/MIPS plot V2492 Cyg is on the Class I-II boundary. 

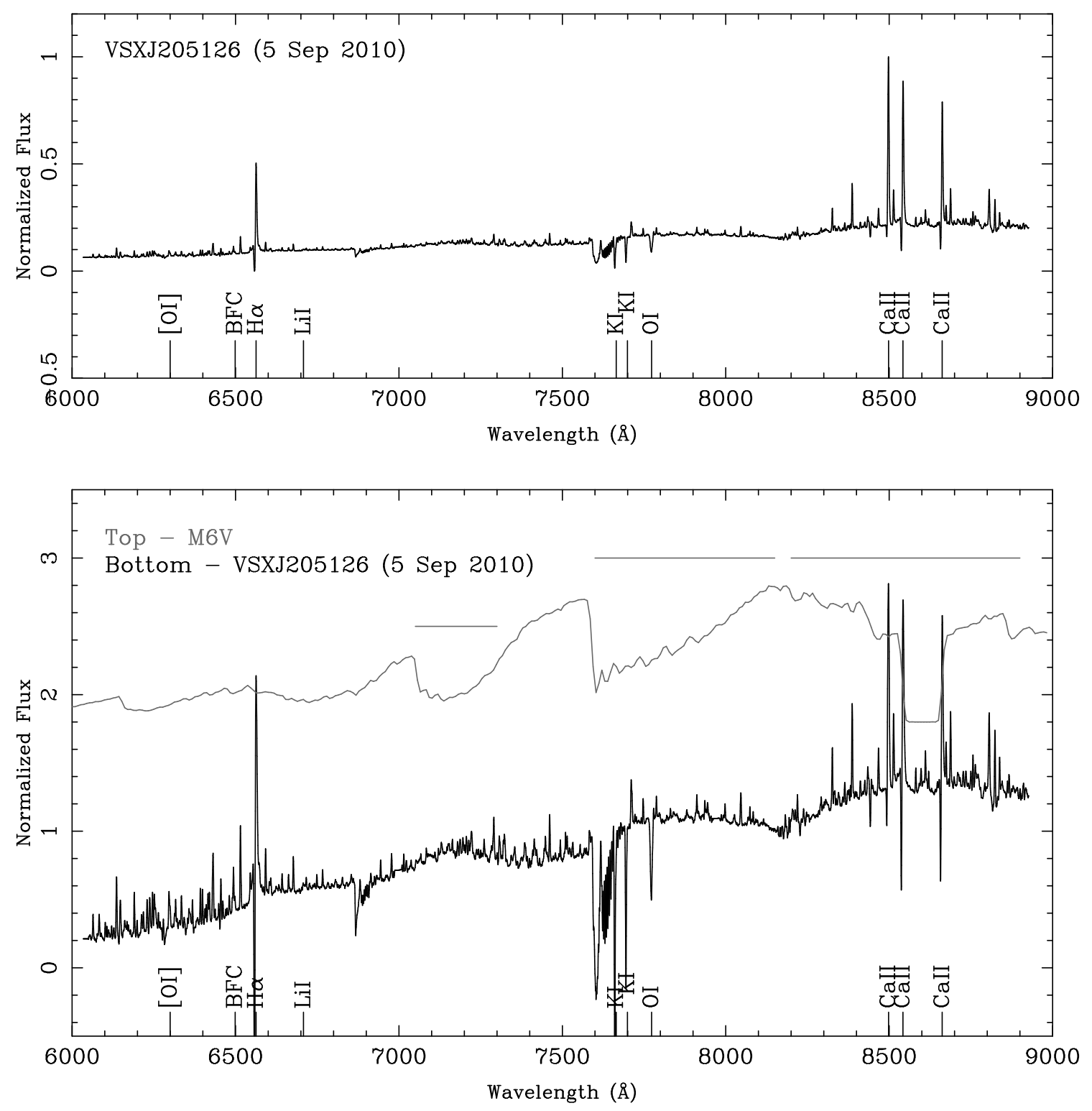

Fig. 9.- Top: The Gemini-North GMOS spectra of V2492 Cyg covering the wavelength range 6000-9000 $\AA$. The source shows $\mathrm{H} \alpha$ and far-red Ca II triplet emission, both with significantly blue-shifted absorption components. Both K I and O I are seen in absorption and many other weaker lines are in emission. BFC refers to the blended Ba II, Fe I, and Ca I lines at $\sim 6500 \AA$. Bottom: The same spectrum of V2492 Cyg but displayed on a $\log _{10}$ scale. Also shown is a spectrum of an M6 V star to show the location of the TiO absorption bands which are in emission in V2492 Cyg. 

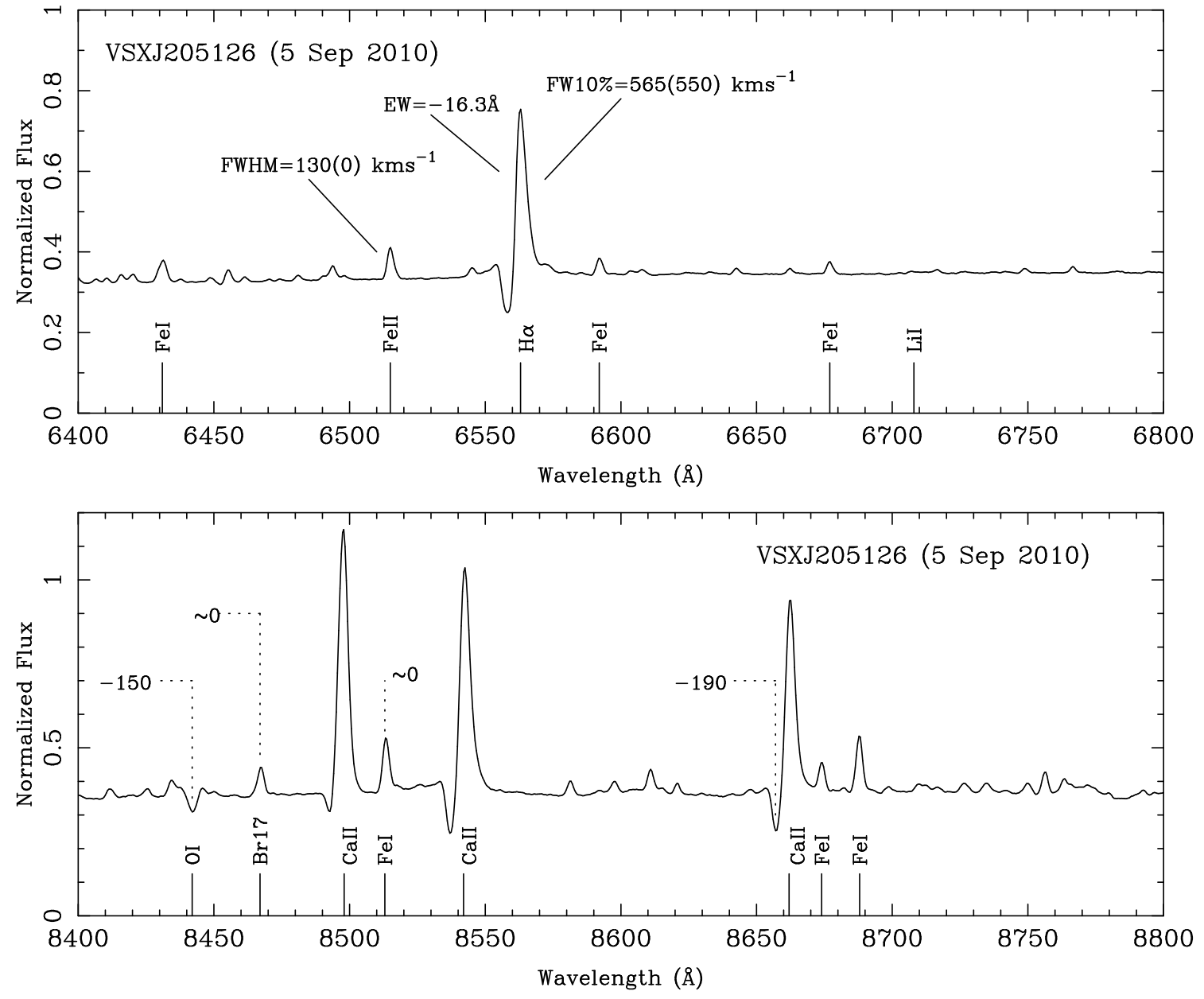

Fig. 10. - Top, the 6400-6800 $\AA$ region of the spectra shown in Figure 9. H $\alpha$ is strongly in emission and clearly shows an associated blue-shifted absorption component creating a $\mathrm{P}$ Cygni profile. The velocity of the minimum absorption is at a blue-shifted velocity of $-216 \mathrm{~km} \mathrm{~s}^{-1}$. The Ba II, Fe I, Ca I blend at $\sim 6500 \AA$ is not present, however, emission lines from Fe I and Fe II are seen. Li I absorption is not present and must be heavily veiled by emission. Bottom, the 8400-8800 $\AA$ region of the spectra shown in Figure 9, This region contains the far-red Ca II triplet lines. V2492 Cyg shows the far-red Ca II triplet lines strongly in emission with blue-shifted absorption components with the center of the absorption occurring at $-190 \mathrm{~km} \mathrm{~s}^{-1}$. 

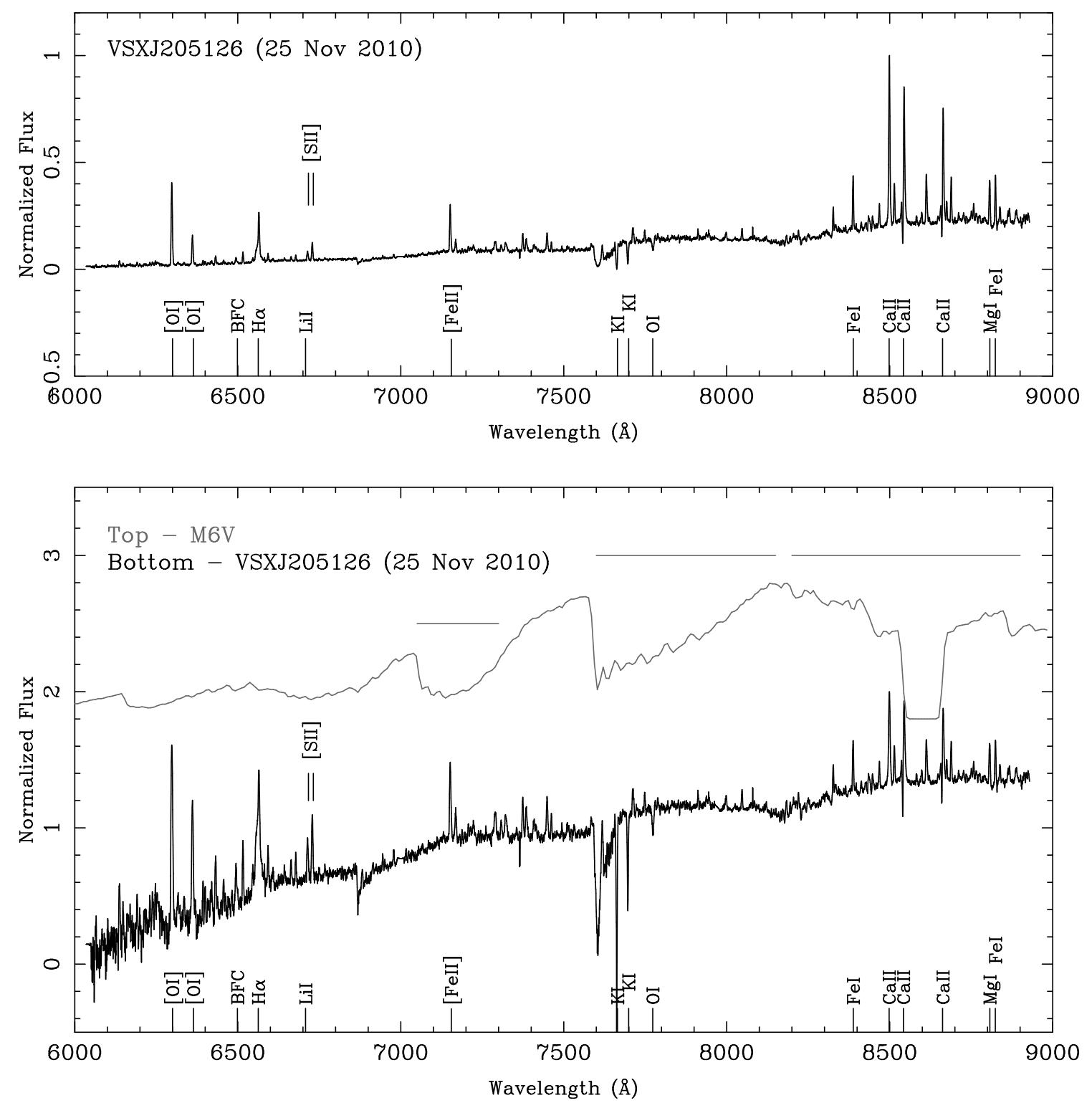

Fig. 11.- Top: The Gemini-North GMOS spectra of V2492 Cyg from UT 2010 November 25 covering the wavelength range 6000-9000 $\AA$. The source shows $\mathrm{H} \alpha$ and far-red Ca II triplet emission with $\mathrm{K} \mathrm{I}$ and $\mathrm{O} \mathrm{I}$ in absorption. BFC refers to the blended Ba II, Fe I, and Ca I lines at $\sim 6500 \AA$. Bottom: The same spectrum of V2492 Cyg but displayed on a $\log _{10}$ scale. Also shown is a spectrum of an M6 V star to show the location of the TiO absorption bands which are in emission in V2492 Cyg. 

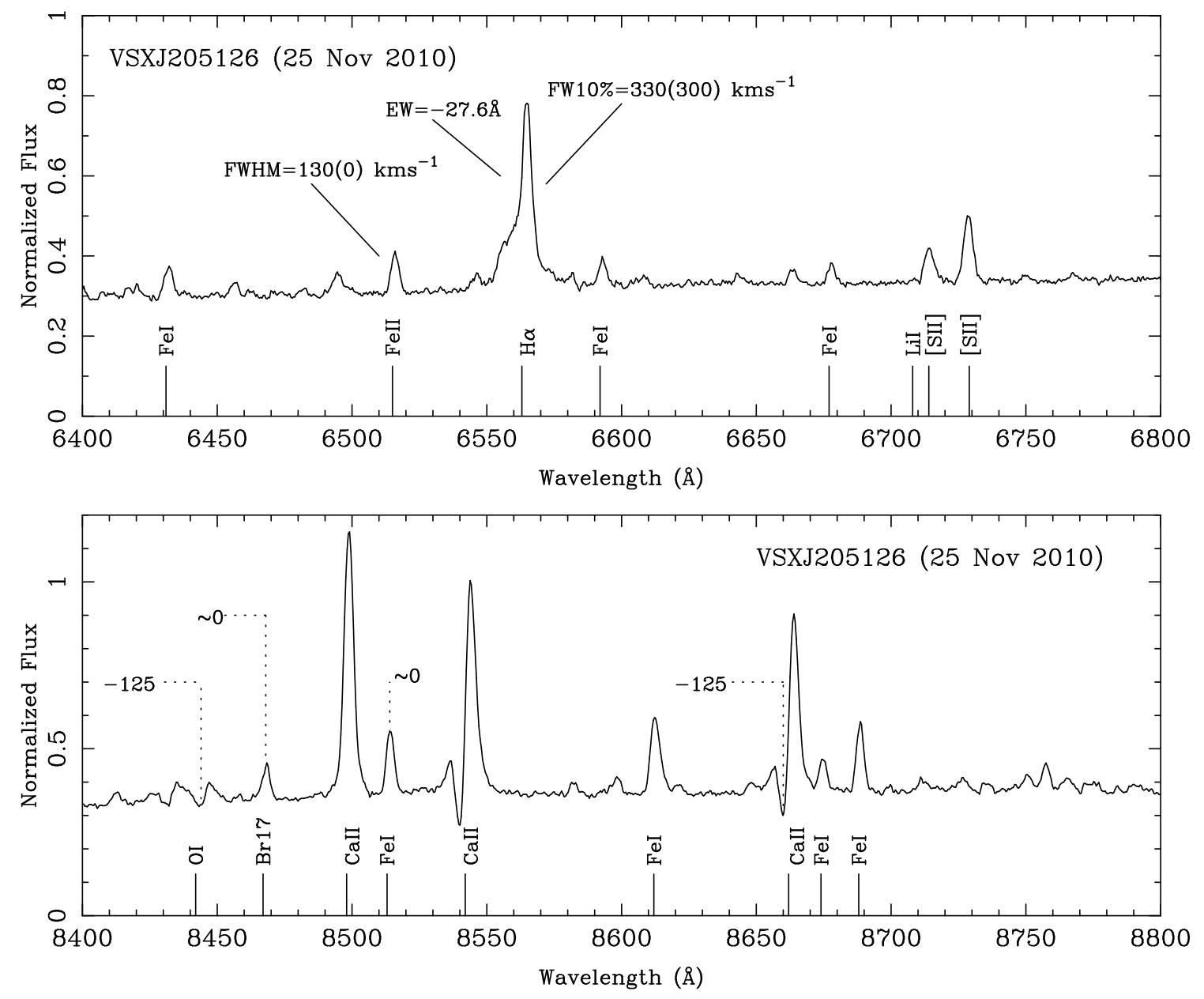

Fig. 12.- Top, the 6400-6800 $\AA$ region of the spectra shown in Figure 11. H $\alpha$ is in emission but the associated blue-shifted absorption component seen on September 5 has disappeared. Bottom, the 8400-8800 $\AA$ region of the spectra shown in Figure 11. This region contains the far-red Ca II triplet lines. V2492 Cyg shows the far-red Ca II triplet lines strongly in emission with blue-shifted absorption components visible on two of the three lines. The center of the absorption trough has decreased from $-190 \mathrm{~km} \mathrm{~s}^{-1}$ on September 5 to $-125 \mathrm{~km} \mathrm{~s}^{-1}$ on UT 2010 November 25. 

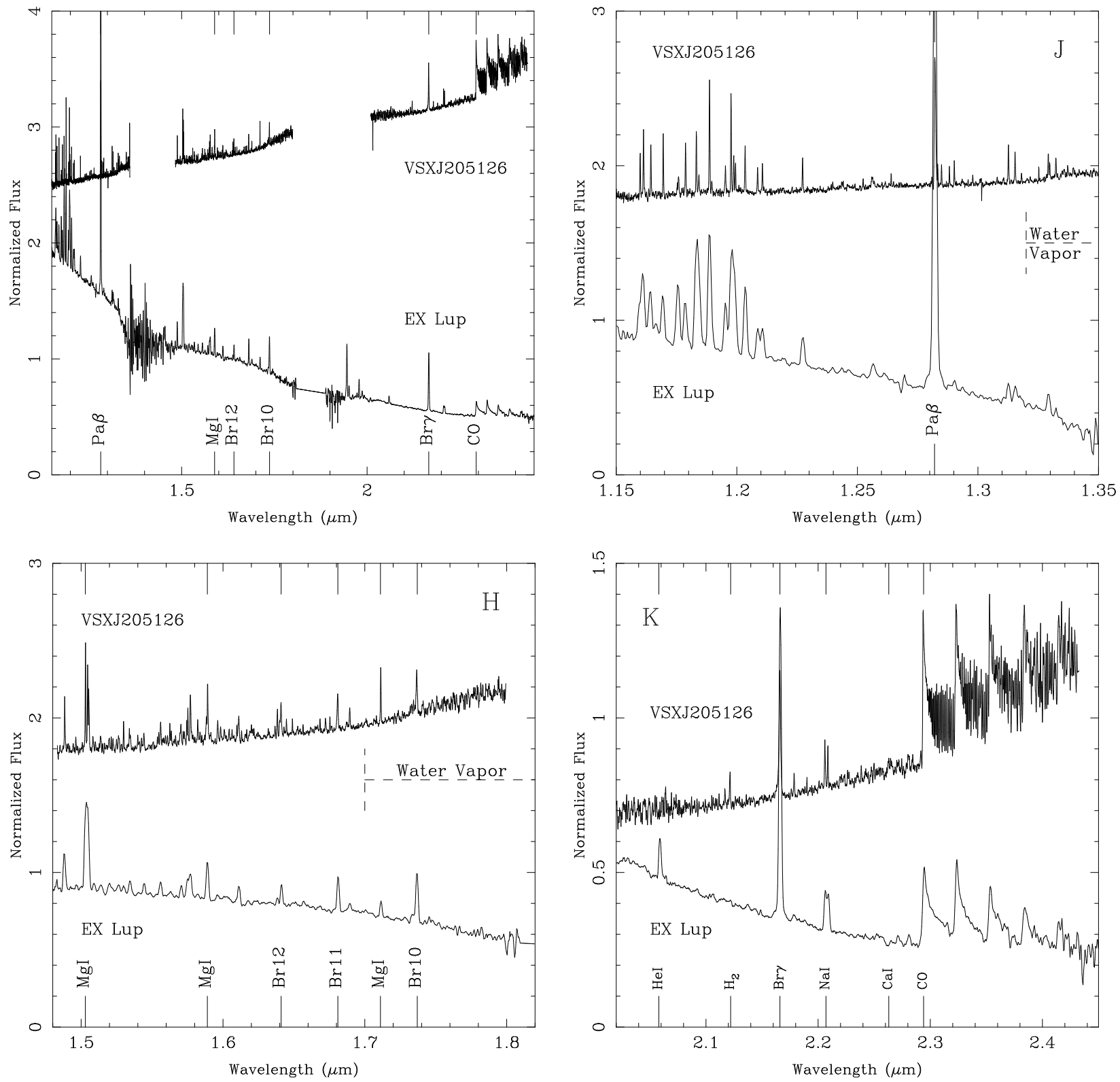

Fig. 13.- NIFS spectra of V2492 Cyg from UT 2010 October 2 and 3. Top-left is the full J, $\mathrm{H}$, and $\mathrm{K}$ band spectra. Top-right, bottom-left, and bottom-right show closer views of the J, H, and $\mathrm{K}$ bands, respectively. For comparison, a lower resolution spectrum of EX Lup ( $\mathrm{R} \sim 1200$ taken during its 2008 outburst using the IRTF SpeX spectrograph) is also shown. Note the very similar spectral structure in both sources including CO overtone emission bandheads, and both $\operatorname{Br} \gamma$ and $\mathrm{Pa} \beta$ strongly in emission. The forest of emission lines from $1.15-1.22 \mu \mathrm{m}$ are predominantly from Fe I. 


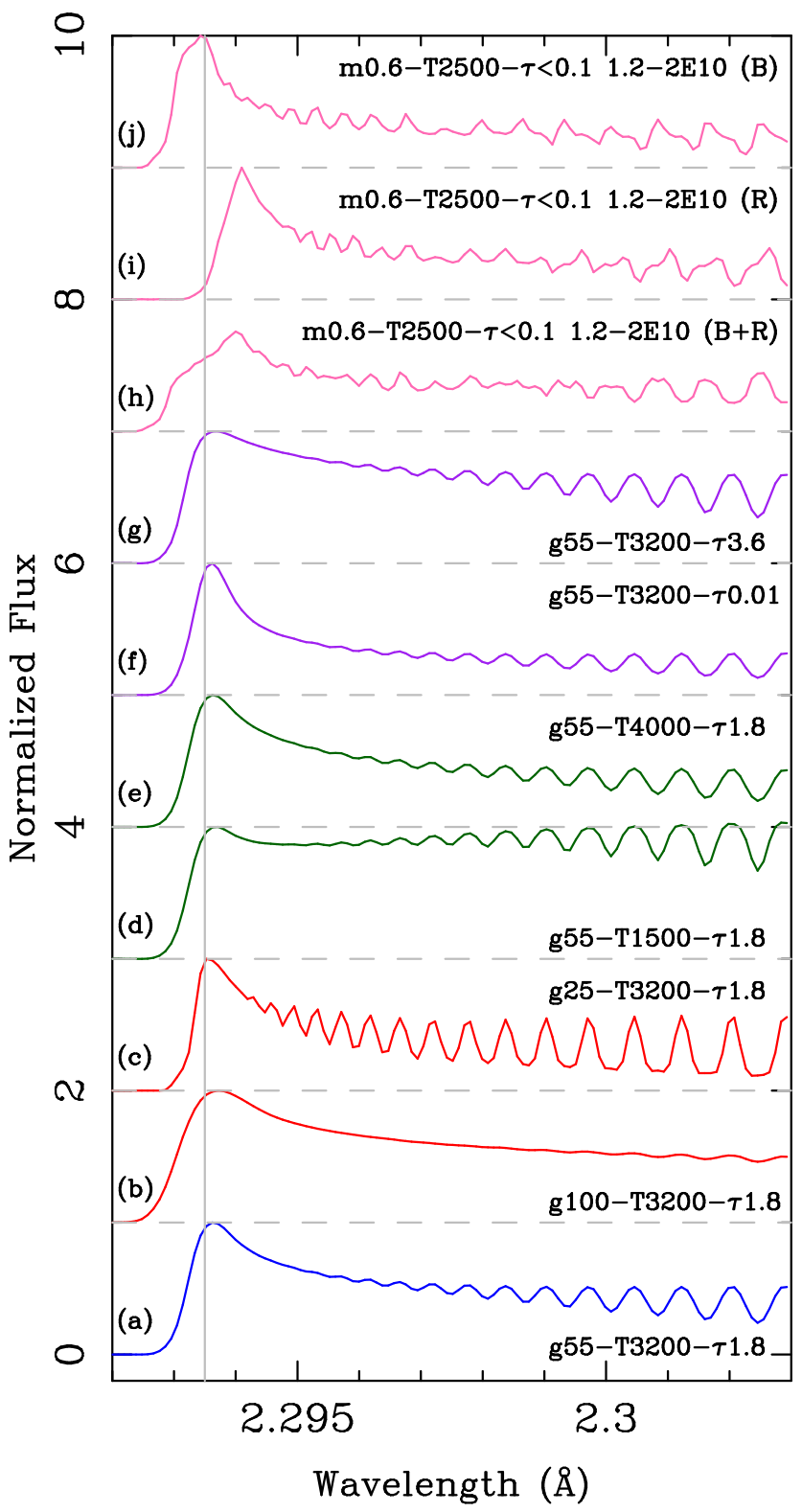

Fig. 14.- A selection of $\mathrm{CO}$ overtone bandhead emission models showing the effect of changing the free parameters. The parameters used to generate the model are shown in each sub-plot. Sub-plot (a, blue) shows the best-fit model to the V2492 Cyg data shown in Figure 15] (e.g. Gaussian velocity broadening $v_{g}=55 \mathrm{~km} \mathrm{~s}^{-1}$, CO gas temperature $\mathrm{T}_{C O}=3200 \mathrm{~K}$, and $\mathrm{CO}$ optical depth $\tau_{C O}=1.8$ ). Sub-plots (b) and (c, both red) show the effect of changing $v_{g}$ to 100 and $25 \mathrm{~km} \mathrm{~s}^{-1}$, respectively. Sub-plots (d) and (e, both green) show the result of changing $\mathrm{T}_{C O}$ to 1500 and $4000 \mathrm{~K}$, respectively. Sub-plots (f) and (g, purple) show the effect of changing $\tau_{C O}$ to 0.01 and 3.6, respectively. Sub-plot (h, pink) shows the changes that occur when a disk velocity profile is used rather than a Gaussian profile. The parameters used here are stellar mass $\mathrm{M}_{*}=0.6 \mathrm{M}_{\odot}, \mathrm{T}_{C O}=2500 \mathrm{~K}, \tau_{C O}=0.1$, and the inner $\left(r_{\text {in }}\right)$ and outer $\left(r_{\text {out }}\right)$ disk radius of 1.2 and $2.0 \times 10^{10} \mathrm{~m}$, respectively, and are the values used in the best-fit EX Lup model shown in Figure 15. Plots (i) and (j, also pink) show the effect of occulting the blue-shifted and red-shifted disk velocities, respectively. 


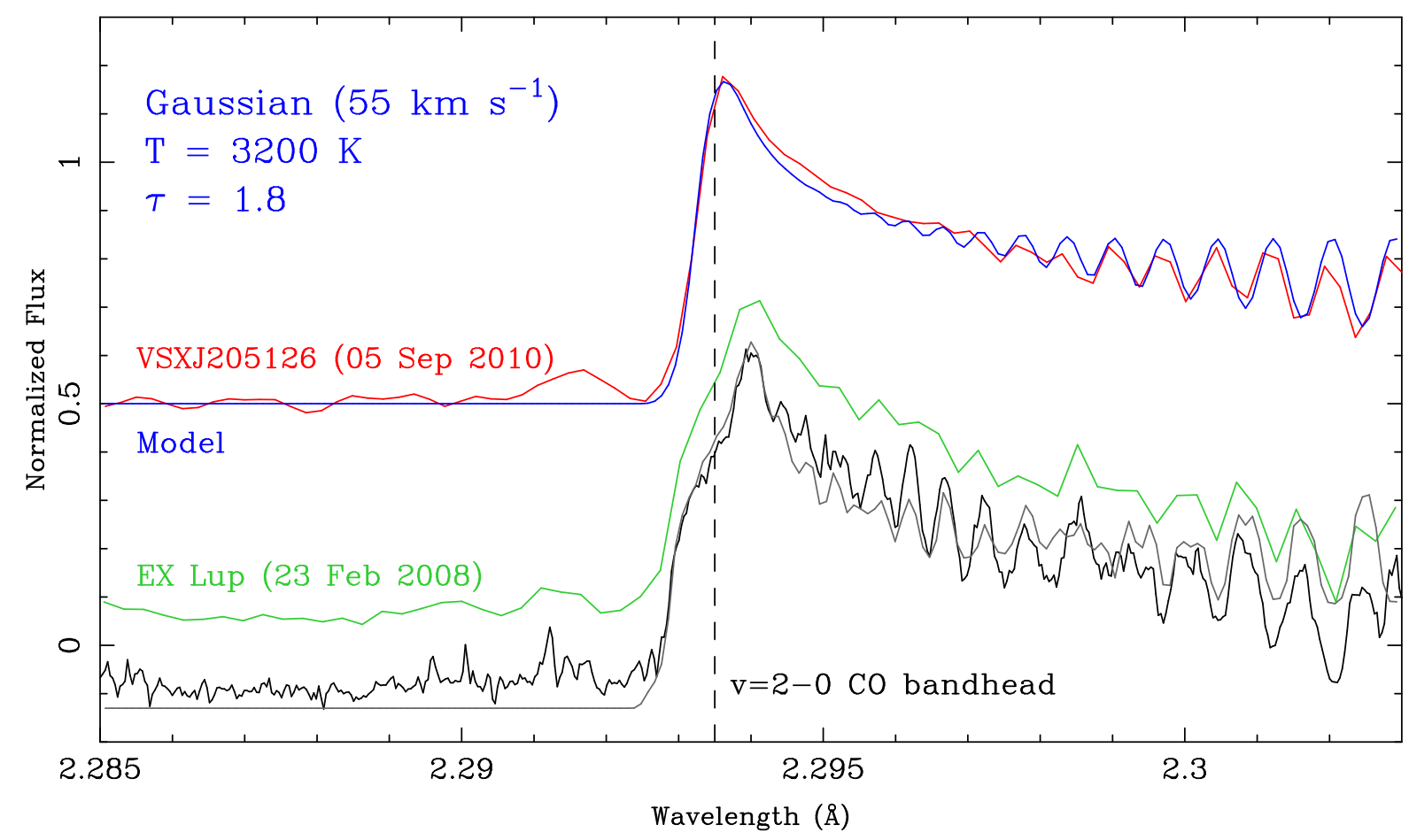

Fig. 15. - The $\mathrm{v}=2-0 \mathrm{CO}$ overtone bandhead emission profile from UT October 2 (red) overlaid by the best-fit model (blue). Also shown are observations and a model for EX Lup during its 2008 extreme outburst (from Aspin et al. 2010). The EX Lup data was taken at higher spectral resolution (black) but was binned to the resolution of the V2492 Cyg spectrum (green) to show that the structure present is still visible in the lower resolution version. The best-fit model to the EX Lup data is also shown (grey). The parameters used to produce the best-fit model to the V2492 Cyg spectrum are shown (top-left). 

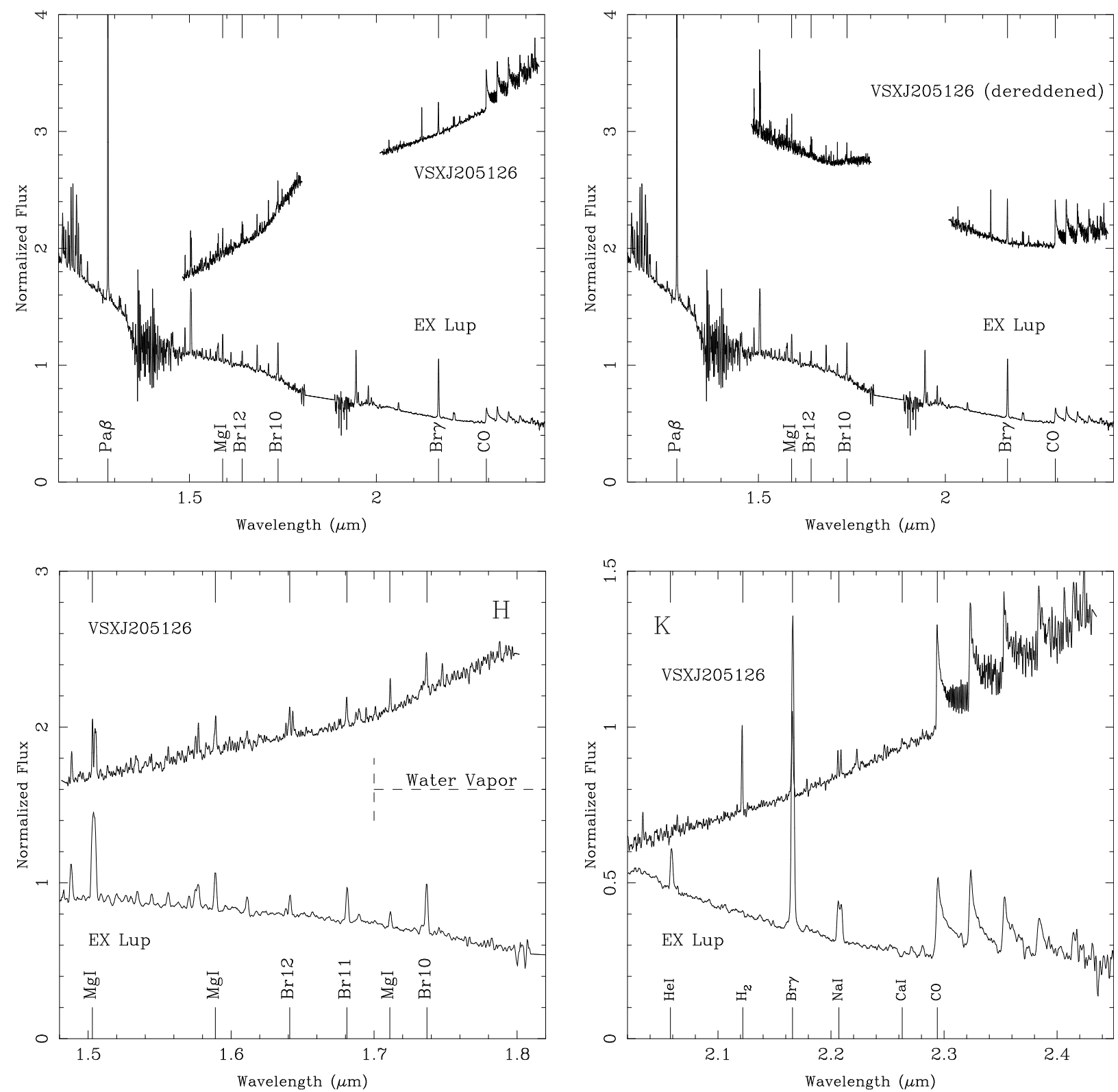

Fig. 16. - NIFS spectra of V2492 Cyg from UT November 26 and 27. Top-left is the full $\mathrm{H}$ and $\mathrm{K}$ band spectra ( $\mathrm{J}$ was not obtained on this date). Bottom-left and bottom-right show closer views of the $\mathrm{H}$, and $\mathrm{K}$ bands, respectively. Again, the spectrum of EX Lup is also shown. In the top-right panel we show a dereddened version of the full $\mathrm{H}$ to $\mathrm{K}$-band spectrum of V2492 Cyg using an $\mathrm{A}_{V}=20$ mags. This demonstrates that dereddening can closely match the continuum slope of EX Lup which has an intrinsic $A_{V}$ of 0-1 mags. 


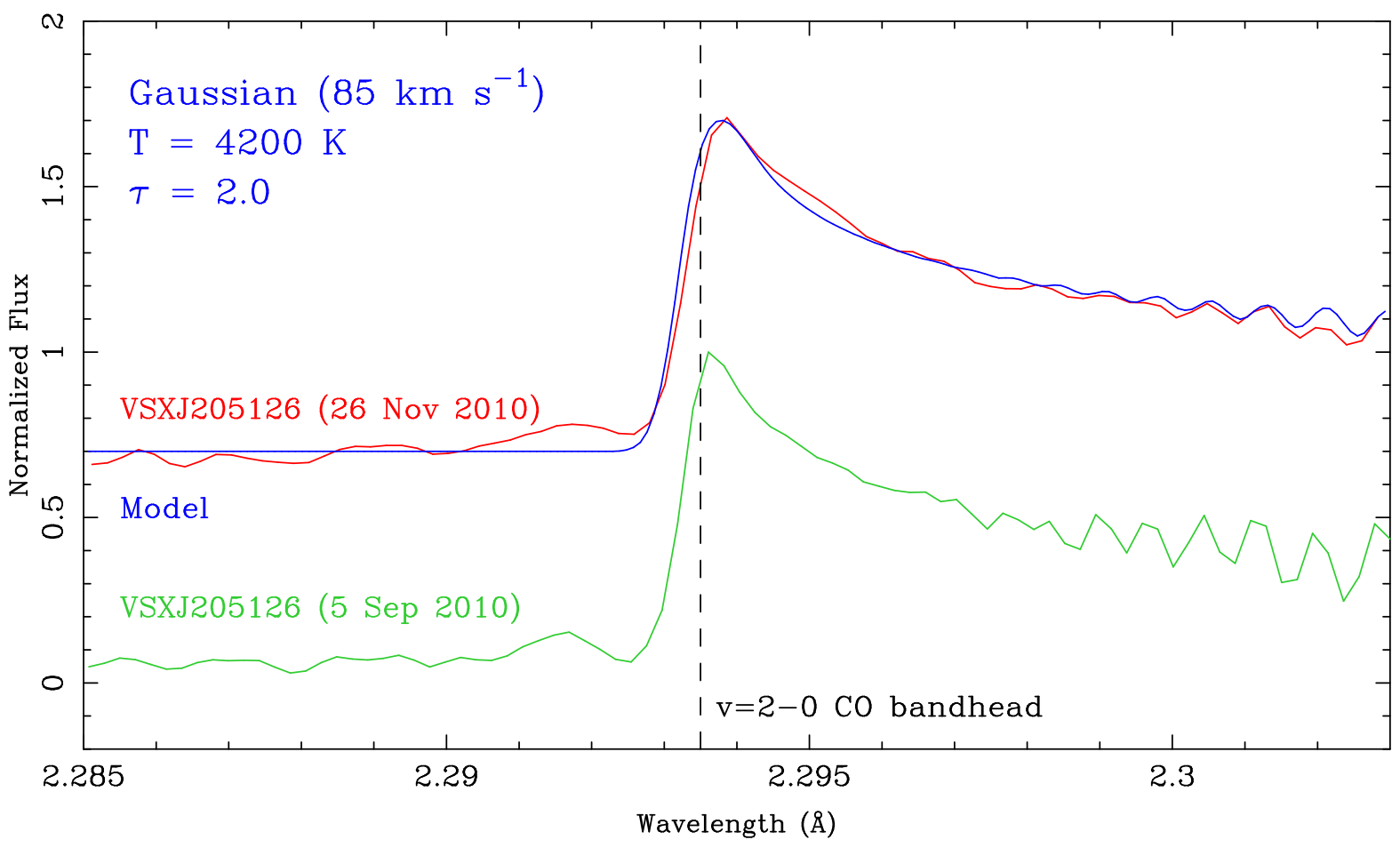

Fig. 17. - The $\mathrm{v}=2-0 \mathrm{CO}$ overtone bandhead emission profile from UT November 26 (red) overlaid by the best-fit model (blue). As in Figure 15, observations (black and green) and best-fit model (grey) for EX Lup are shown. The parameters used to produce the best-fit model to the V2492 Cyg spectrum are shown (top-left). 


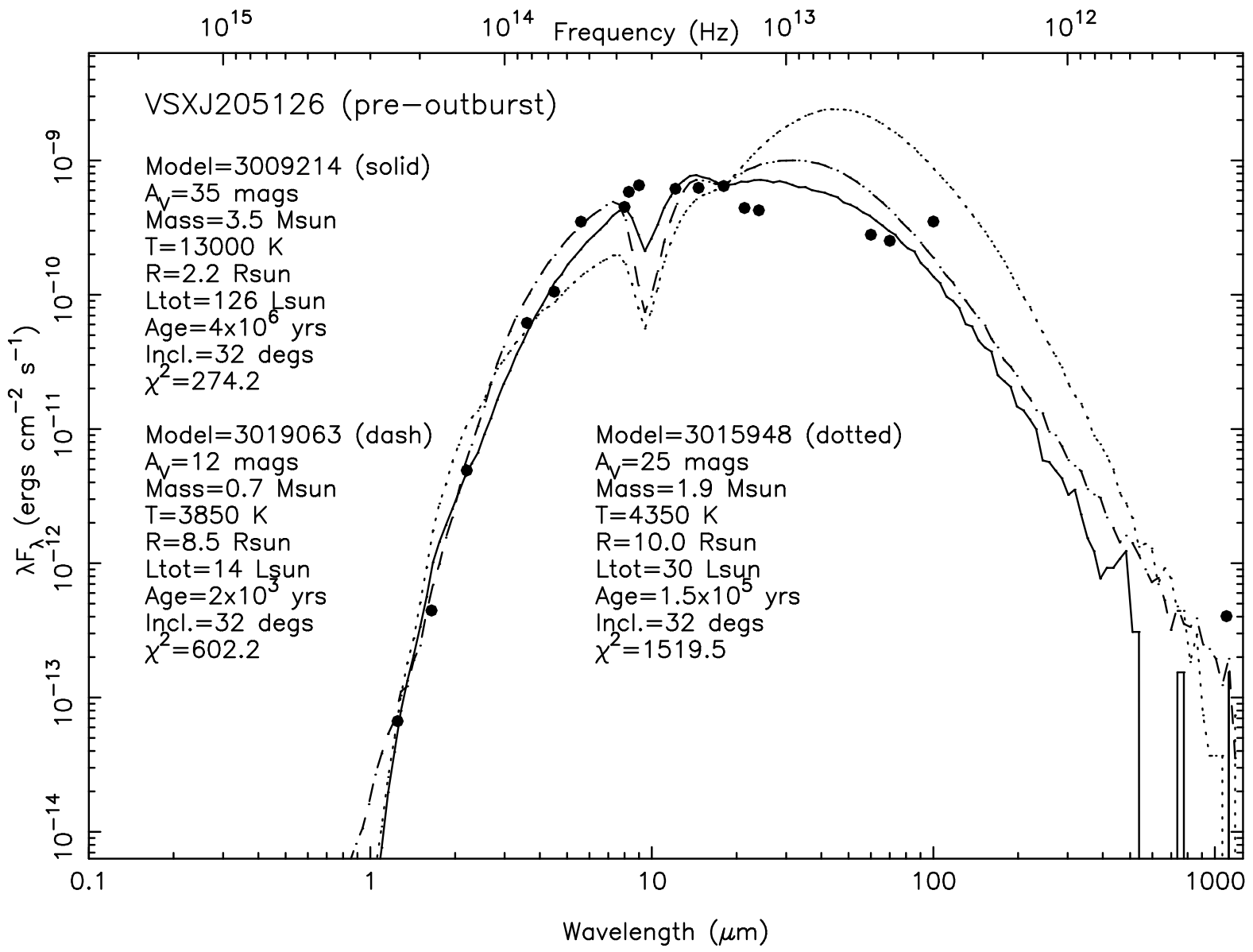

Fig. 18. - Pre-outburst spectral energy distribution (SED) for V2492 Cyg. The J, H, K' fluxes are from the UKIDSS database, the 8.28, 9.0, 12.13, 14.65, 18.0, and $21.34 \mu \mathrm{m}$ fluxes are from $M S X 6 C$, while the 60 and $100 \mu \mathrm{m}$ fluxes are from IRAS. Three models from Robitaille et al. (2007) are overlaid on the data. The solid line shows the overall best fit. The dashed line shows the best fit with $\mathrm{M}_{*}$ constrained to be $<2 \mathrm{M}_{\odot}$. The dotted line shows the best fit constrained with both $\mathrm{M}_{*}<2 \mathrm{M}_{\odot}$ and $10^{5}<\mathrm{t}_{*}<10^{7} \mathrm{yrs}$. 